\title{
Nonlinear multidimensional cosmological models with form fields: Stabilization of extra dimensions and the cosmological constant problem
}

\author{
U. Günther* \\ Gravitationsprojekt, Mathematische Physik I, Institut für Mathematik, Universität Potsdam, Am Neuen Palais 10, PF 601553, \\ D-14415 Potsdam, Germany \\ and Max-Planck-Institut für Gravitationsphysik, Albert-Einstein-Institut, Am Mühlenberg 1, D-14476 Golm, Germany \\ P. Moniz ${ }^{\dagger}$ \\ Departamento de Física, Universidade da Beira Interior, Rua Marquês D’Ávila e Bolama, 6200 Covilhã, Portugal
}

A. Zhuk

Department of Physics, University of Odessa, 2 Dvoryanskaya St., Odessa 65100, Ukraine

and Max-Planck-Institut für Gravitationsphysik, Albert-Einstein-Institut, Am Mühlenberg 1, D-14476 Golm, Germany

(Received 2 March 2003; published 12 August 2003)

\begin{abstract}
We consider multidimensional gravitational models with a nonlinear scalar curvature term and form fields in the action functional. In our scenario it is assumed that the higher dimensional spacetime undergoes a spontaneous compactification to a warped product manifold. Particular attention is paid to models with quadratic scalar curvature terms and a Freund-Rubin-like ansatz for solitonic form fields. It is shown that for certain parameter ranges the extra dimensions are stabilized. In particular, stabilization is possible for any sign of the internal space curvature, the bulk cosmological constant, and of the effective four-dimensional cosmological constant. Moreover, the effective cosmological constant can satisfy the observable limit on the dark energy density. Finally, we discuss the restrictions on the parameters of the considered nonlinear models and how they follow from the connection between the D-dimensional and the four-dimensional fundamental mass scales.
\end{abstract}

DOI: 10.1103/PhysRevD.68.044010

PACS number(s): 04.50. $+\mathrm{h}, 11.25 . \mathrm{Mj}, 98.80 . \mathrm{Jk}$

\section{INTRODUCTION}

Two of the most intriguing problems of modern cosmology are the problem of additional dimensions and the cosmological constant problem (CCP). The first problem follows from theories which unify different fundamental interactions with gravity, such as $\mathbf{M}$ or string theory [1], and which have their most consistent formulation in spacetimes with more than four dimensions. The problem can be naturally formulated as the following question: if we live in a multidimensional spacetime, why do we not observe the extra dimensions? Within the "old" Kaluza-Klein (KK) framework and the early $\left(E_{8} \times E_{8}\right)$-heterotic string phenomenology, the question is answered by assuming the extra dimensions are so small (i.e., with a characteristic size $r$ between the Planck and the Fermi scales $10^{-33} \mathrm{~cm} \leqslant r$ $\lesssim 10^{-17} \mathrm{~cm}$ ) that they are not accessable by present-day collider experiments. New concepts with the possibility for rich phenomenology opened up with the uncovering of $D$ - branes by Polchinski [2] in 1995. In "brane-world" scenarios of the Universe the usual four-dimensional physics with its $S U(3) \times S U(2) \times U(1)$ standard model (SM) fields is localized on a three-dimensional space-like hypersurface

\footnotetext{
*Electronic address: u.guenther@fz-rossendorf.de

Present address: Research Center Rossendorf, P.O. Box 510119, D-01314 Dresden, Germany.

${ }^{\dagger}$ Electronic address: pmoniz@dfisica.ubi.pt; also at Centra-IST,

Rua Rovisco Pais, 1049 Lisboa, Portugal.

‡Electronic address: zhuk@paco.net
}

(our world-brane), whereas the gravitational field propagates in the whole (bulk) spacetime. Depending on the concrete scenario, there are different types of masking of the additional dimensions. Whereas in Arkani-Hamed-DvaliDimopoulos (ADD) models [3-5] the extra dimensions are curled up to sizes smaller than $10^{-2} \mathrm{~cm}$, so that they are in agreement with present table-top Cavendish-type tests of gravity [6], they can be infinite in the Randall-Sundrum II (RS II) [7] and Dvali-Gabadadze-Porrati (DGP) [8] models. In the latter models, the four-dimensionality of low-energy physics is achieved by inducing appropriate effective gravitational potentials on the world-brane. In addition to their interesting phenomenology, brane-world models provide a possible resolution of the hierarchy problem. In ADD-type models this is due to the connection between the Planck scale $M_{P l(4)}$ and the fundamental scale $M_{*\left(4+D^{\prime}\right)}$ of the four-dimensional and the $\left(4+D^{\prime}\right)$-dimensional spacetimes, respectively:

$$
M_{P l(4)}^{2} \sim V_{D^{\prime}} M_{*\left(4+D^{\prime}\right)}^{2+D^{\prime}} .
$$

$V_{D^{\prime}}$ denotes the volume of the compactified $D^{\prime}$ extra dimensions. It was realized in [3-5] that localizing the SM fields on a 3-brane allows one to lower $M_{*\left(4+D^{\prime}\right)}$ down to the electroweak scale $M_{E W} \sim 1 \mathrm{TeV}$ without contradiction with present observations. Therefore, the compactification scale of the internal space can be of order

$$
r \sim V_{D^{\prime}}^{1 / D^{\prime}} \sim 10^{\left(32 / D^{\prime}\right)-17} \mathrm{~cm} .
$$


With $M_{E W} \sim 1 \mathrm{TeV}$, physically acceptable values correspond to $D^{\prime} \geqslant 3$ [3] (for astrophysical and cosmological bounds see, e.g., [9]; experimental bounds from table-top Cavendishtype experiments are given in [6]), and for $D^{\prime}=3$ one arrives at a submillimeter compactification scale $r \sim 10^{-5} \mathrm{~mm}$ of the internal space. If we shift $M_{*\left(4+D^{\prime}\right)}$ to $30 \mathrm{TeV}$, as suggested in [9] (see also [10]), then the $D^{\prime}=2$ case satisfies all aforementioned bounds and leads to $r \sim 10^{-3}-10^{-2} \mathrm{~mm}$. In order not to exclude this $D^{\prime}=2$ with its largest possible compactification scale $r$, we assume that the fundamental scale $M_{*\left(4+D^{\prime}\right)}$ can be of order $30 \mathrm{TeV}$. Additionally, the geometry in the ADD approach is assumed to be factorizable as in a standard Kaluza-Klein model. That is, the topology is the direct product of a nonwarped external spacetime manifold and internal space manifolds with warp factors which depend on the external coordinates. ${ }^{1}$

According to observations, the internal space should be static or nearly static at least from the time of primordial nucleosynthesis (otherwise the fundamental physical constants would vary, see, e.g., $[12,13])$. This means that at the present evolutionary stage of the Universe the compactification scale of the internal space should either be stabilized and trapped at the minimum of some effective potential, or it should be slowly varying (similar to the slowly varying cosmological constant in the quintessence scenario [14]). In both cases, small fluctuations over stabilized or slowly varying compactification scales (conformal scales/geometrical moduli) are possible.

Stabilization of extra dimensions (moduli stabilization) in models with large extra dimensions (ADD-type models) has been considered in a number of papers (see, e.g., Refs. $[5,15-21]) .^{2}$ In the corresponding approaches, a product topology of the $\left(4+D^{\prime}\right)$-dimensional bulk spacetime was constructed from Einstein spaces with scale (warp) factors depending only on the coordinates of the external fourdimensional component. As a consequence, the conformal excitations had the form of massive scalar fields living in the external spacetime. Within the framework of multidimensional cosmological models (MCM), such excitations were investigated in [22-24] where they were called gravitational excitons. Later, since the ADD compactification approach these geometrical moduli excitations are known as radions $[5,16]$.

Most of the aforementioned papers are devoted to the stabilization of large extra dimension in theories with a linear multidimensional gravitational action. String theory suggests that the usual linear Einstein-Hilbert action should be ex-

\footnotetext{
${ }^{1}$ The $\mathrm{M}$ theory inspired RS scenarios $[7,11]$ use a nonfactorizable geometry with $D^{\prime}=1$. Here, the four-dimensional spacetime is warped with a factor $\widetilde{\Omega}$ which depends on the extra dimension and Eq. (1) is modified as $M_{P l(4)} \sim \widetilde{\Omega}^{-1} M_{E W}$. In our paper we concentrate on the factorizable geometry of ADD-type models.

${ }^{2}$ In most of these papers, moduli stabilization was considered without regard to the energy-momentum localized on the brane so that the dynamics of the multidimensional universe was mainly defined by the energy-momentum of the bulk matter. A brane matter contribution was taken into account, e.g., in [21].
}

tended with higher-order nonlinear curvature terms. In a previous paper [25] we considered a simplified model with a multidimensional Lagrangian of the form $L=f(R)$, where $f(R)$ is an arbitrary smooth function of the scalar curvature. Without connection to stabilization of the extra dimensions, such models (four-dimensional as well as multidimensional ones) were considered, e.g., in Refs. [26-28]. There, it was shown that the nonlinear models are equivalent to models with linear gravitational action plus a minimally coupled scalar field with self-interaction potential. In [25], we advanced this equivalence towards investigating the stabilization problem for extra dimensions. Particular attention was paid to models with quadratic scalar curvature terms. It was shown that for certain parameter ranges, the extra dimensions are stabilized if the internal spaces have negative constant curvature. In this case, the four-dimensional effective cosmological constant $\Lambda_{\text {eff }}$ as well as the bulk cosmological constant $\Lambda_{D}$ become negative. As a consequence, the homogeneous and isotropic external space is asymptotically $\mathrm{AdS}_{4}$. Because the considered nonlinear model is a pure geometrical one (only with a bare cosmological constant $\Lambda_{D}$ as an exotic matter source included) the equivalent linear model contains only a minimally coupled scalar field as bulk matter. The null energy condition (NEC) $T_{a b} N^{a} N^{b} \geqslant 0$ for this field reads $T_{a b} N^{a} N^{b}=\left(N^{a} \partial_{a} \phi\right)^{2} \geqslant 0$ (with $N$ a future directed null vector) and is satisfied only marginally when the internal spaces are completely stabilized and the scalar field is frozen out. Moreover, the weak energy condition (WEC) $T_{a b} W^{a} W^{b} \geqslant 0$ (with $W$ a future directed timelike vector) is violated in this case because the energy density $\rho$ of the scalar field is negative definite $\rho<0$. As a result, the aforementioned parameters (the internal space scalar curvatures, $\Lambda_{D}$ and $\left.\Lambda_{e f f}\right)$ are negative in the case of stabilized internal spaces (see also $[17,20,24]$ ).

However, a negative cosmological constant leads to a deceleration of the Universe instead of to an accelerated expansion, as recent observational data indicate. According to these data our Universe is dominated by a dark energy component with negative pressure. For example, from observations of the clusters of galaxies it follows that the energy density of the matter components which can clump in various structures is significantly undercritical. But, the position of the first acoustic peak in the angular power spectrum of the cosmic microwave background (CMB) radiation implies that the Universe is, on large scales, nearly flat. In other words, the energy density in the Universe is very close to the critical value. Thus, there must exist a homogeneously distributed exotic (dark) energy component [29]. This observation is in agreement with the conclusion following from the Hubble diagram of type Ia supernovae (SN-Ia) at high redshifts, which also indicate that our Universe currently undergoes an accelerated expansion. Under the assumption of flatness, using the data of the CMB anisotropy measurements, high redshift SN-Ia observations, and from local cluster abundances, the authors of Ref. [30] found a constraint on the equation of state parameter $\omega_{Q}=P / \rho<-0.85$ at $68 \%$ confidence level. They concluded that this result is in perfect agreement with the $\omega_{Q}=-1$ cosmological constant case and 
gives no support to a quintessential field scenario with $\omega_{Q}$ $>-1$. Results obtained in [31] also favor $\omega_{Q} \approx-1$ at the present epoch.

In Ref. [25] we already indicated that the effective cosmological constant can be shifted from negative values to positive ones by including into the nonlinear model matter fields which satisfy the NEC. In the present paper, we demonstrate this effect explicitly by endowing the extra dimensions with real-valued solitonic form fields [32]. Such fields naturally arise as Ramond-Ramond (RR) form fields in type II string theory and $\mathrm{M}$ theory. Within a generalized FreundRubin setting [33] their influence on the evolutionary dynamics of the Universe has been considered, e.g., in Refs. [34-37] and due to its simplicity we adopt this ansatz here for the stability analysis of our nonlinear model. From Eqs. (9), (10) below, it can be easily seen that the real-valued form fields satisfy the NEC as well as the WEC. However, the strong energy condition (SEC) is violated in our model by the cosmological constant. ${ }^{3}$ The presence of two types of fields in our equivalent linear model - the minimally coupled scalar field (which satisfies the NEC only marginally and which can violate the WEC) and the form fields (which satisfy both of these conditions) — leads to a rich and interesting picture of stable configurations with various sign combinations for the allowed cosmological constants as well as for the constant curvatures of the internal space. Beside stability regions with negative four-dimensional effective cosmological constant $\Lambda_{\text {eff }}<0$ the parameter space contains also regions with $\Lambda_{e f f}>0$ which can ensure an accelerated expansion of the Universe.

As mentioned at the very beginning of the Introduction, there still remains the problem of the incredible smallness of the cosmological constant [39,40]. Moreover, it is completely unclear why its energy density is comparable with the energy density of matter just at the present time (the cosmic coincidence problem). Modern reviews on the cosmological constant problem can be found, for example, in [29,41,42]. In our paper, we show that for stabilized internal spaces a small positive cosmological constant of the external (our) space can arise from ADD- and KK-type multidimensional models. We demonstrate that the smallness of the effective cosmological constant can follow from a natural parameter choice of the considered nonlinear ansatz. Unfortunately, the extremely small value of the observed cosmological constant requires a very strong fine tuning of the parameters.

The paper is structured as follows. The general setup of our model is given in Sec. II. There we make the geometry of the spacetime manifold explicit-endowing the internal space with the structure of a warped product of $n$ factor spaces (due to spontaneous compactification). Furthermore, we specify the generalized Freund-Rubin ansatz for the form fields. In Sec. III, we present a detailed analysis of the stabilization problem for a model with one internal space. The main results are summarized and discussed in the concluding Sec. IV.

${ }^{3}$ For a critical discussion of the different ECs, we refer the reader to [38].

\section{GENERAL SETUP}

We consider a $D=\left(4+D^{\prime}\right)$-dimensional nonlinear gravitational theory with action

$$
\begin{aligned}
S= & \frac{1}{2 \kappa_{D}^{2}} \int_{M} d^{D} x \sqrt{|\bar{g}|} f(\bar{R}) \\
& -\frac{1}{2} \int_{M} d^{D} x \sqrt{|\bar{g}|} \sum_{i=1}^{n} \frac{1}{d_{i} !}\left(F^{(i)}\right)^{2},
\end{aligned}
$$

where $f(\bar{R})$ is an arbitrary smooth function with mass dimension $O\left(\mathrm{~m}^{2}\right)$ ( $m$ has the unit of mass) of the scalar curvature $\bar{R}=R[\bar{g}]$ constructed from the $D$-dimensional metric $\bar{g}_{a b}(a, b=1, \ldots, D)$,

$$
\kappa_{D}^{2}=8 \pi / M_{*\left(4+D^{\prime}\right)}^{2+D^{\prime}}
$$

denotes the $D$-dimensional gravitational constant (subsequently, we assume that $\left.M_{*\left(4+D^{\prime}\right)} \sim M_{E W}\right)$. In action (3), $F^{(i)}=F_{m_{i} n_{i} \ldots q_{i}}^{(i)}, i=1, \ldots, n$ is an antisymmetric tensor field of rank $d_{i}$ (a $d_{i}$-form-field strength) with indices from an index set $s_{(i)}=\left\{m_{i}: \max \left(m_{i}\right)-\min \left(m_{i}\right)=d_{i}\right\}$, where $m_{i}, n_{i}, \ldots, q_{i} \in s_{(i)}$. For simplicity, we suppose that the index sets $s_{(i)}, s_{(j)}$ of tensors $F^{(i)}, F^{(j)}$ with $i \neq j$ contain no common elements as well as no indices corresponding to the coordinates of the $D_{0}$-dimensional external spacetime (usually $\left.D_{0}=4\right)$. Additionally, we assume that for the sum of the ranks holds $\sum_{i=1}^{n} d_{i}=D-D_{0}:=D^{\prime}$. Obviously, this model can be generalized to tensor configurations $F^{(i)}, F^{(j)}$ with intersecting (overlapping) index sets. In this case explicit field configuration can be obtained, e.g., when the indices satisfy special overlapping rules [36]. Such a generalization is beyond the scope of the present paper. Furthermore, we assume in our subsequent considerations that the index sets $m_{i}, n_{i}, \ldots, q_{i} \neq 0$ do not contain the coordinates of the external spacetime $M_{0}$ and, hence, the field strengths $F^{(i)}$ can be associated with a magnetic (solitonic) $p$-brane system located in the extra dimensions as discussed, e.g., in Refs. $[32,35,36]$.

The equation of motion for the gravitational sector of Eq. (3) reads

$$
f^{\prime} \bar{R}_{a b}-\frac{1}{2} f \bar{g}_{a b}-\bar{\nabla}_{a} \bar{\nabla}_{b} f^{\prime}+\bar{g}_{a b} \square f^{\prime}=\kappa_{D}^{2} T_{a b}[F, \bar{g}]
$$

where $a, b=1, \ldots, D, f^{\prime}=d f / d \bar{R}, \bar{R}_{a b}=R_{a b}[\bar{g}]$, and $\bar{R}$ $=R[\bar{g}] . \bar{\nabla}_{a}$ and $\square$ denote the covariant derivative and the Laplacian with respect to the metric $\bar{g}_{a b}$,

$$
\bar{\square}=\square[\bar{g}]=\bar{g}^{a b} \bar{\nabla}_{a} \bar{\nabla}_{b}=\frac{1}{\sqrt{|\bar{g}|}} \partial_{a}\left(\sqrt{|\bar{g}|} \bar{g}^{a b} \partial_{b}\right)
$$

Equation (5) can be rewritten in the form 


$$
\begin{aligned}
& f^{\prime} \bar{G}_{a b}+\frac{1}{2} \bar{g}_{a b}\left(\bar{R} f^{\prime}-f\right)-\bar{\nabla}_{a} \bar{\nabla}_{b} f^{\prime}+\bar{g}_{a b} \square f^{\prime} \\
& =\kappa_{D}^{2} T_{a b}[F, \bar{g}],
\end{aligned}
$$

where $\bar{G}_{a b}=\bar{R}_{a b}-\frac{1}{2} \bar{R} \bar{g}_{a b}$, and its trace

$$
(D-1) \square f^{\prime}=\frac{D}{2} f-f^{\prime} \bar{R}+\kappa_{D}^{2} T[F, \bar{g}]
$$

can be considered as a connection between $\bar{R}$ and $f$. The energy momentum tensor (EMT) $T_{a b}[F, \bar{g}]$ is defined in the standard way as

$$
\begin{aligned}
T_{a b}[F, \bar{g}] & \equiv \frac{1}{\sqrt{|\bar{g}|}} \frac{\delta\left(\sqrt{|\bar{g}|} \sum_{i=1}^{n} \frac{1}{d_{i} !}\left(F^{(i)}\right)^{2}\right)}{\delta \bar{g}^{a b}} \\
& =\sum_{i=1}^{n} T_{a b}\left[F^{(i)}, \bar{g}\right],
\end{aligned}
$$

where

$$
\begin{aligned}
T_{a b}\left[F^{(i)}, \bar{g}\right]= & \frac{1}{d_{i} !}\left(-\frac{1}{2} \bar{g}_{a b} F_{m_{i} n_{i} \cdots q_{i}}^{(i)} F^{(i)} m_{i} n_{i} \cdots q_{i}\right. \\
& \left.+d_{i} F_{a n_{i} \cdots q_{i}}^{(i)} F_{b}^{(i) n_{i} \cdots q_{i}}\right) .
\end{aligned}
$$

For the trace of this tensor we obtain

$$
T[F, \bar{g}]=\sum_{i=1}^{n} T\left[F^{(i)}, \bar{g}\right]
$$

with

$$
T\left[F^{(i)}, \bar{g}\right]=\frac{2 d_{i}-D}{2\left(d_{i} !\right)} F_{m_{i} n_{i} \cdots q_{i}}^{(i)} F^{(i) m_{i} n_{i} \cdots q_{i}} .
$$

The field strengths $F^{(i)}$ satisfy the equations of motion

$$
\begin{gathered}
F^{(i) m_{i} n_{i} \cdots q_{i}} q_{i}=0 \Leftrightarrow \frac{1}{\sqrt{|\bar{g}|}}\left(\sqrt{|\bar{g}|} F^{(i) m_{i} n_{i} \cdots q_{i}}\right)_{q_{i}}=0, \\
i=1, \cdots, n
\end{gathered}
$$

and the Bianchi identities

$$
F_{\left[m_{i} n_{i} \cdots q_{i}, a\right]}^{(i)}=0, i=1, \ldots, n .
$$

Following Refs. [26-28], we perform a conformal transformation

$$
g_{a b}=\Omega^{2} \bar{g}_{a b}
$$

with

$$
\Omega=\left[f^{\prime}(\bar{R})\right]^{1 /(D-2)}
$$

and reduce the nonlinear gravitational theory to a linear one with an additional scalar field. This transformation is well defined for $f^{\prime}(\bar{R})>0$ (concerning the case $f^{\prime} \leqslant 0$, see footnote 6 ). The equivalence of the theories can be easily proven with the help of the auxiliary formulas

$$
\begin{aligned}
& \square= \Omega^{-2}\left[\bar{\square}+(D-2) \bar{g}^{a b} \Omega^{-1} \Omega_{, a} \partial_{b}\right], \\
& \square=\Omega^{2}\left[\square-(D-2) g^{a b} \Omega^{-1} \Omega_{, a} \partial_{b}\right], \\
& R_{a b}=\bar{R}_{a b}+\frac{D-1}{D-2}\left(f^{\prime}\right)^{-2} \bar{\nabla}_{a} f^{\prime} \bar{\nabla}_{b} f^{\prime}-\left(f^{\prime}\right)^{-1} \bar{\nabla}_{a} \bar{\nabla}_{b} f^{\prime} \\
&-\frac{1}{D-2} \bar{g}_{a b}\left(f^{\prime}\right)^{-1} \bar{\square} f^{\prime},
\end{aligned}
$$

and

$$
\begin{aligned}
R= & \left(f^{\prime}\right)^{2 /(2-D)}\left\{\bar{R}+\frac{D-1}{D-2}\left(f^{\prime}\right)^{-2} \bar{g}^{a b} \partial_{a} f^{\prime} \partial_{b} f^{\prime}\right. \\
& \left.-2 \frac{D-1}{D-2}\left(f^{\prime}\right)^{-1} \bar{\square} f^{\prime}\right\} .
\end{aligned}
$$

Defining the scalar $\phi$ by the relation

$$
f^{\prime}=\frac{d f}{d \bar{R}}:=e^{A \phi}>0, \quad A:=\sqrt{\frac{D-2}{D-1}}
$$

and making use of Eqs. (17)-(19), Eqs. (7) and (8) can be rewritten as

$$
G_{a b}=\kappa_{D}^{2} T_{a b}[F, \phi, g]+T_{a b}[\phi, g]
$$

and

$$
\begin{aligned}
\square \phi= & \frac{1}{\sqrt{(D-1)(D-2)}} \exp \left(\frac{-D}{\sqrt{(D-1)(D-2)}} \phi\right) \\
& \times\left(\frac{D}{2} f-f^{\prime} \bar{R}\right)+\frac{1}{\sqrt{(D-1)(D-2)}} \kappa_{D}^{2} T[F, \phi, g] .
\end{aligned}
$$

The EMTs read

$$
\begin{aligned}
T_{a b}[\phi, g]= & \phi_{, a} \phi_{, b}-\frac{1}{2} g_{a b} g^{m n} \phi_{, m} \phi_{, n}-\frac{1}{2} g_{a b} \\
& \times \exp \left(\frac{-D}{\sqrt{(D-1)(D-2)}} \phi\right)\left(\bar{R} f^{\prime}-f\right),
\end{aligned}
$$

$$
T_{a b}[F, \phi, g]=\sum_{i=1}^{n} \exp \left(\frac{2 d_{i}-D}{\sqrt{(D-1)(D-2)}} \phi\right) T_{a b}\left[F^{(i)}, g\right],
$$


and

$$
T[F, \phi, g]=\sum_{i=1}^{n} \exp \left(\frac{2 d_{i}-D}{\sqrt{(D-1)(D-2)}} \phi\right) T\left[F^{(i)}, g\right],
$$

where $T_{a b}\left[F^{(i)}, g\right], T\left[F^{(i)}, g\right]$ are given by replacing $\bar{g} \rightarrow g$ in Eqs. (10), (12). The indices of the field strengths $F^{(i)}$ are now raised and lowered with the metric $g$.

The equations of motion (13) for $F^{(i)}$ transform to

$$
\begin{gathered}
\frac{1}{\sqrt{|g|}}\left[\sqrt{|g|} \exp \left(\frac{2 d_{i}-D}{\sqrt{(D-1)(D-2)}} \phi\right) F^{(i) m_{i} n_{i} \cdots q_{i}}\right]_{, q_{i}}=0, \\
i=1, \ldots, n,
\end{gathered}
$$

whereas the Bianchi identities (14) do not change.

It can be easily checked that Eqs. (21), (22), and (26) are the equations of motion for the action

$$
\begin{aligned}
S= & \frac{1}{2 \kappa_{D}^{2}} \int_{M} d^{D} x \sqrt{|g|}\left\{R[g]-g^{a b} \phi_{, a} \phi_{, b}-2 U(\phi)-\kappa_{D}^{2}\right. \\
& \left.\times \sum_{i=1}^{n} \frac{1}{d_{i} !} \exp \left(\frac{2 d_{i}-D}{\sqrt{(D-1)(D-2)}} \phi\right) F_{m_{i} n_{i} \cdots q_{i}}^{(i)} F^{(i) m_{i} n_{i} \cdots q_{i}}\right\},
\end{aligned}
$$

where

$$
\begin{gathered}
U(\phi):=\frac{1}{2} e^{-B \phi}\left[\bar{R}(\phi) e^{A \phi}-f(\bar{R}(\phi))\right], \\
B:=\frac{D}{\sqrt{(D-1)(D-2)}}
\end{gathered}
$$

and Eq. (20) is used to express $\bar{R}$ as a function of $\phi: \bar{R}$ $=\bar{R}(\phi)$. The scalar field $\phi$ is the result and the carrier of the curvature nonlinearity of the original theory ${ }^{4}$ (3). Correspondingly, Eq. (22) has a twofold interpretation. It is the equation of motion for the field $\phi$ and at the same time it can be considered as a constraint equation following from the reduction of the nonlinear theory (3) to the linear one (27). Furthermore, we note that in the linear theory (27) the form fields are nonminimally coupled with the nonlinearity field $\phi$. [A minimal coupling occurs only for a model with $n$ $=1, d_{1}=D_{0}$, where according to Eq. (12) the trace of the form field EMT vanishes.] A comparison of the action functional with Eq. (24) shows that the last term in Eq. (27) coincides with the expression for the energy density $-T_{0}^{0}[F, \phi, g]$ of the solitonic form field (due to $F_{0 n_{i} \cdots q_{i}}^{(i)}$ $\equiv 0$ by the definition of $F^{(i)}$ ).

Let us consider what happens if, in some way, the scalar field $\phi$ tends asymptotically to a constant: $\phi \rightarrow \phi_{0}$ [precisely

\footnotetext{
${ }^{4}$ Thus, for brevity, we shall refer to the field $\phi$ as a nonlinearity scalar field.
}

this situation should hold when the internal space undergoes a (freezing) stabilization]. From Eq. (20) we see that in this limit the nonlinearity in Eq. (3) disappears: $f(\bar{R}) \approx c_{1}(\bar{R}$ $\left.-\bar{R}_{0}\right)+f\left(\bar{R}_{0}\right) \equiv c_{1} \bar{R}+c_{2}, \quad$ where $\quad c_{1}:=f^{\prime}\left(\bar{R}_{0}\right)=\exp \left(A \phi_{0}\right)$, $\bar{R}_{0} \equiv \bar{R}\left(\phi_{0}\right)$, and $-c_{2} /\left(2 c_{1}\right)$ plays the role of a cosmological constant. In the case of homogeneous and isotropic spacetime manifolds, linear purely geometrical theories with a constant $\Lambda$ term necessarily imply an (A)dS geometry so that the manifolds are Einstein spaces. In our model, the additional form fields destroy this asymptotical behavior. Instead, we obtain from Eqs. (8) and (7)

$$
\begin{aligned}
\bar{R} \rightarrow & -\frac{D}{D-2} \frac{c_{2}}{c_{1}}-\frac{1}{c_{1}} \frac{2}{D-2} \kappa_{D}^{2} \\
& \times \sum_{i=1}^{n} \frac{2 d_{i}-D}{2\left(d_{i} !\right)}\left(F^{(i)}\right)_{\frac{2}{g}}^{2}
\end{aligned}
$$

and

$$
\begin{aligned}
\bar{R}_{a b} \rightarrow & {\left[-\frac{1}{D-2} \frac{c_{2}}{c_{1}}-\frac{1}{c_{1}} \frac{2}{D-2} \kappa_{D}^{2}\right.} \\
& \left.\times \sum_{i=1}^{n} \frac{d_{i}-1}{2\left(d_{i} !\right)}\left(F^{(i)}\right) \frac{2}{g}\right] \bar{g}_{a b} \\
& +\frac{1}{c_{1}} \kappa_{D}^{2} \sum_{i=1}^{n} \frac{2 d_{i}}{2\left(d_{i} !\right)}\left(F_{a n_{i} \cdots q_{i}}^{(i)} F_{b}^{(i) n_{i} \cdots q_{i}}\right)_{g}^{-},
\end{aligned}
$$

where the form field product

$$
\left(F^{(i)}\right)_{\frac{g}{g}}^{2}=F_{m_{i} n_{i} \cdots q_{i}}^{(i)} F^{(i) m_{i} n_{i} \cdots q_{i}}
$$

is defined with respect to the metric $\bar{g}$. For a form field, which asymptotically tends to a constant, the scalar curvature and the Ricci tensor also approach constant values. But whereas $\bar{R}$ and $R$ are asymptotically connected by the relation [see Eqs. (19), (21), and (29)]

$$
R \rightarrow c_{1}^{-2 /(D-2)} \bar{R},
$$

the Ricci tensor $\bar{R}_{a b}$ will not be proportional to the metric $\bar{g}_{a b}$ and, hence, the space will not be Einsteinian. This is in obvious contrast to a nonlinear model of purely geometrical type [25] where the stabilization will result in an asymptotical $(\mathrm{A}) \mathrm{dS}_{D}$ spacetime.

In the rest of the paper we consider for simplicity a toy model $^{5}$ with a quadratic curvature term,

$$
f(\bar{R})=\bar{R}+\alpha \bar{R}^{2}-2 \Lambda_{D},
$$

\footnotetext{
${ }^{5}$ For considerations on higher-order corrections to the gravity sector of $\mathrm{M}$ string theory, we refer the reader to $[28,43]$.
} 
where the parameter $\alpha$ has dimension $O\left(m^{-2}\right)$. For this model we obtain

$$
f^{\prime}(\bar{R})=1+2 \alpha \bar{R}=e^{A \phi} \Leftrightarrow \bar{R}=\frac{1}{2 \alpha}\left(e^{A \phi}-1\right)
$$

and

$$
U(\phi)=\frac{1}{2} e^{-B \phi}\left[\frac{1}{4 \alpha}\left(e^{A \phi}-1\right)^{2}+2 \Lambda_{D}\right] .
$$

The condition ${ }^{6} f^{\prime}>0$ implies $1+2 \alpha \bar{R}>0$. In the limit $\alpha$ $\rightarrow 0$, the nonlinearity is switched off and the linear theory is recovered. Correspondingly, it holds $f^{\prime} \rightarrow 1$ with the implication $c_{1}=1, \phi_{0} \rightarrow 0$ so that also $\bar{R} \rightarrow R$ [in accordance with Eq. (32)] and $U(\phi \rightarrow 0) \rightarrow \Lambda_{D}$. The corresponding region of weak nonlinearity is defined by the condition $\alpha \bar{R}=e^{A \phi}-1$ $\ll 1$.

Furthermore, we assume that the multidimensional spacetime manifold undergoes a spontaneous compactification

$$
M \rightarrow M=M_{0} \times M_{1} \times \cdots \times M_{n}
$$

in accordance with the block-orthogonal structure of the field strength $F$, and that the form fields $F^{(i)}$, each nested in its own $d_{i}$-dimensional factor space $M_{i}$, respect a generalized Freund-Rubin ansatz [33] (see also [34-37]). This allows us to perform a dimensional reduction of our model along the lines of Refs. [22-25,45,46]. The factor spaces $M_{i}$ are then Einstein spaces with metrics $g^{(i)} \equiv e^{2 \beta^{i}(x)} \gamma^{(i)}$ which depend only through the warp factors $a_{i}(x):=e^{2 \beta^{i}(x)}$ on the coordinates $x$ of the external spacetime $M_{0}$. For the corresponding scalar curvatures holds

$$
R\left[\gamma^{(i)}\right]=\lambda^{i} d_{i} \equiv R_{i} \sim k r_{i}^{-2},
$$

${ }^{6}$ Obviously, the conformal transformation (15),(16) becomes singular when $f^{\prime}(\bar{R})$ vanishes. The transformation itself can be extended from the $f^{\prime}>0$ branch to the $f^{\prime}<0$ branch with the help of an ansatz [27] $\Omega=\left|f^{\prime}(\bar{R})\right|^{1 /(D-2)}$ and a corresponding redefinition of the nonlinearity field $\phi: e^{A \phi}=\left|f^{\prime}\right|$. As a result, one obtains an action functional for the $f^{\prime}<0$ branch which differs from action (27) for $f^{\prime}>0$ in its total sign and in the relative signs of the single terms as well as in the potential $U(\phi)$. Most important, for a fixed sign of the Einstein-Hilbert term the kinetic term of the nonlinearity field has the correct sign, whereas the kinetic terms of additional matter fields (in our case the form fields) have the wrong relative sign. This leads to a set of equations of motions which differ from Eqs. (21),(22). For details we refer to [27]. Unfortunately, the sign switch occurs for all additional matter fields simultaneously and it is not controllable for some selected fields separately. Otherwise, it could have provided a natural mechanism for the generation of a phantom energy component with equation of state parameter $\omega_{Q}$ $<-1$ (and a corresponding super-acceleration of the observable Universe) in the sense of [44]. where $k=0, \pm 1$. The effective radii $r_{i}$ are defined from the volumes $^{7}$

$$
V_{d_{i}} \equiv \int_{M_{i}} d^{d_{i}} y \sqrt{\left|\gamma^{(i)}\right|} \sim r_{i}^{d_{i}}, \quad i=1, \ldots, n,
$$

where $V_{d_{i}}$ have dimensions $O\left(m^{-d_{i}}\right)$. For technical details of the dimensional reduction with subsequent conformal transformation from the intermediate Brans-Dicke frame to the Einstein frame of the external spacetime $M_{0}$, as well as for notations we refer to Sec. III of our previous paper [25]. According to the corresponding approach the warped product of Einstein spaces leads to a scalar curvature $\bar{R}$ which depends only on the coordinate $x$ of the $D_{0}$-dimensional external spacetime $M_{0}: \bar{R}[\bar{g}]=\bar{R}(x)$. This implies that the nonlinearity field $\phi$ is also a function only of $x$ : $\phi=\phi(x)$.

In the rest of the present section we restrict our attention to the form-field components which were not part of the purely geometrical model of Ref. [25]. We choose them in the generalized Freund-Rubin ansatz as

$$
\begin{gathered}
F_{m_{i} n_{i} \cdots q_{i}}^{(i)}=\sqrt{2} \sqrt{\left|g^{(i)}\right|} \varepsilon_{m_{i} n_{i} \cdots q_{i}} f^{(i)}(x), \\
F^{(i) m_{i} n_{i} \cdots q_{i}}=\left(\sqrt{2} / \sqrt{\left|g^{(i)}\right|}\right) \varepsilon^{m_{i} n_{i} \cdots q_{i}} f^{(i)}(x) .
\end{gathered}
$$

For the Levi-Civita symbol $\varepsilon_{m_{i} n_{i} \cdots q_{i}}$ we use conventions where for Riemann spaces hold $\varepsilon_{m_{i} n_{i} \cdots q_{i}}=\varepsilon^{m_{i} n_{i} \cdots q_{i}}$ and $\varepsilon_{m_{i} n_{i} \cdots q_{i}} \varepsilon^{m_{i} n_{i} \cdots q_{i}}=d_{i}$ !. It can be easily seen that the ansatz (39) satisfies Eq. (26) (because $\phi$ and $f$ depend only on $x$ and the $\sqrt{\left|\gamma^{(i)}\right|}$ factors cancel). The Bianchi identities (14) reduce to the equations

$$
\frac{\partial\left[a_{i}^{d_{i}}(x) f^{(i)}(x)\right]}{\partial x^{\mu}}=0
$$

with solutions

$$
f^{(i)}(x)=\frac{f_{i}}{a_{i}^{d_{i}}}
$$

\footnotetext{
${ }^{7}$ The volumes are well defined for positive curvature spaces $(k$ $=+1)$. For compact negative and zero curvature spaces $(k=$ $-1,0)$, i.e., compact hyperbolic spaces (CHSs) $M_{i}=H^{d_{i}} / \Gamma_{i}$ and tori $T_{j}=R^{d_{j}} / \Gamma_{j}$, we interpret them as scaled volumes of the corresponding fundamental domains ("elementary cells") $V_{d_{i}} \sim r_{i}^{d_{i}}$ $\times V_{F D(i)}$ (see, e.g., [47] and references therein). Here $H^{d_{i}}, R^{d_{j}}$ are hyperbolic and flat universal covering spaces, and $\Gamma_{i}, \Gamma_{j}$ are appropriate discrete groups of isometries. Furthermore, we assume for the scale factors of the metrics $\gamma^{i} \sim r_{i} \hat{\gamma}^{i}$ with $\hat{\gamma}^{i}$ scaled in such a way that $V_{F D(i)} \sim O(1)$. Thus, the volume $V_{d_{i}}$ is mainly defined by $r_{i}$. In all three cases $(k= \pm 1,0)$, the limit $r_{i} \rightarrow \infty$ results in an effective decompactification of the internal space with $V_{d_{i}} \rightarrow \infty$. In accordance with Eq. (37), this means that the positive and negative constant curvature spaces flatten: $R_{d_{i}} \rightarrow 0$. Clearly, for compact Ricci-flat spaces holds $R_{d_{i}} \equiv 0$ by definition and without relation to the compactification scale of the torus.
} 
and $f_{i} \equiv$ const. We choose the warp factors $a_{i}$ dimensionless so that the constants $f_{i}^{2}$ have dimension $O\left(m^{4+D^{\prime}}\right)$ and $\kappa_{D}^{2} f_{i}^{2} \sim O\left(m^{2}\right)$. With Eq. (41) the energy density of the solitonic form field, and correspondingly the last term in action (27), reads

$$
\begin{aligned}
-T_{0}^{0}[F, \phi, g]= & \frac{1}{2} \sum_{i=1}^{n} \frac{1}{d_{i} !} \exp \left(\frac{2 d_{i}-D}{\sqrt{(D-1)(D-2)}} \phi\right) \\
& \times F_{m_{i} n_{i} \cdots q_{i}}^{(i)} F^{(i) m_{i} n_{i} \cdots q_{i}} \\
= & \sum_{i=1}^{n} \exp \left(\frac{2 d_{i}-D}{\sqrt{(D-1)(D-2)}} \phi\right) \frac{f_{i}^{2}}{a_{i}^{2 d_{i}}} \\
:= & \rho(\beta, \phi)
\end{aligned}
$$

where for real form fields $f_{i}^{2} \geqslant 0$. Again we see that for models with $n=1$ and $d_{1}=D_{0}$ this energy density decouples from the nonlinearity scalar field $\phi: \rho\left(\beta^{1}, \phi\right) \rightarrow \rho\left(\beta^{1}\right)$.

In the case of a freezing stabilization of the internal spaces with $\beta^{i}=0$, we obtain the nonzero components of the asymptotic Ricci tensor (30) as

$$
\begin{gathered}
\bar{R}_{\mu \nu} \rightarrow \vartheta \bar{g}_{\mu \nu}, \\
\bar{R}_{m_{i} n_{i}} \rightarrow\left(\vartheta+\frac{2}{c_{1}} \kappa_{D}^{2} f_{i}^{2} \Omega_{0}^{2 d_{i}}\right) \bar{g}_{m_{i} n_{i}},
\end{gathered}
$$

where

$$
\vartheta:=-\frac{1}{D-2} \frac{c_{2}}{c_{1}}-\frac{1}{c_{1}} \frac{2}{D-2} \kappa_{D}^{2} \sum_{j=1}^{n}\left(d_{j}-1\right) f_{j}^{2} \Omega_{0}^{2 d_{j}}
$$

and $\Omega_{0}=\left(e^{A \phi_{0}}\right)^{1 /(D-2)}$. Thus, the asymptotic multidimensional spacetime is built up from Einstein-space blocks, but is itself a non-Einsteinian space due to the additional term in Eq. (44).

\section{STABILIZATION OF THE INTERNAL SPACE}

Without loss of generality, ${ }^{8}$ we consider in the present section a model with only one $d_{1}$-dimensional internal space. After dimensional reduction and subsequent conformal transformation to the Einstein frame (along the lines of Ref. [25]), the action functional (27) reads

$$
\begin{aligned}
S= & \frac{1}{2 \kappa_{0}^{2}} \int_{M_{0}} d^{D_{0}} x \sqrt{\left|\tilde{g}^{(0)}\right|}\left\{R\left[\tilde{g}^{(0)}\right]-\widetilde{g}^{(0) \mu \nu} \partial_{\mu} \varphi \partial_{\nu} \varphi\right. \\
& \left.-\widetilde{g}^{(0) \mu \nu} \partial_{\mu} \phi \partial_{\nu} \phi-2 U_{e f f}(\varphi, \phi)\right\},
\end{aligned}
$$

where

\footnotetext{
${ }^{8}$ The difference between a general model with $n>1$ internal spaces and the particular one with $n=1$ consists in an additional diagonalization of the geometrical moduli excitations.
}

$$
\varphi:=-\sqrt{\frac{d_{1}(D-2)}{D_{0}-2}} \beta^{1}
$$

and

$$
\kappa_{0}^{2}:=\frac{\kappa_{D}^{2}}{V_{d_{1}}}
$$

denotes the $D_{0}$-dimensional (four-dimensional) gravitational constant. If we take the electroweak scale $M_{E W}$ and the Planck scale $M_{P l(4)}$ as fundamental ones for $D$-dimensional [see Eq. (4)] and four-dimensional spacetimes $\left(\kappa_{0}^{2}=8 \pi / M_{P l(4)}^{2}\right)$, respectively, then we reproduce Eqs. (1) and (2) with $D^{\prime}=d_{1}$. A stable compactification of the internal space $M_{1}$ is ensured when its scale factor $\varphi$ is frozen at one of the minima of the effective potential,

$$
\begin{aligned}
U_{e f f}(\varphi, \phi)= & \exp \left(2 \varphi \sqrt{\frac{d_{1}}{(D-2)\left(D_{0}-2\right)}}\right) \\
& \times\left[-\frac{1}{2} R_{1} \exp \left(2 \varphi \sqrt{\frac{D_{0}-2}{d_{1}(D-2)}}\right)\right. \\
& \left.+U(\phi)+\kappa_{D}^{2} \rho(\varphi, \phi)\right] .
\end{aligned}
$$

The value of the effective potential at the minimum plays the role of the effective $D_{0}$-dimensional cosmological constant: $\left.U_{e f f}\right|_{\min } \equiv \Lambda_{e f f}$. The potential $U(\phi)$ of the nonlinearity scalar field is given by Eq. (35) and the energy density (42) of the solitonic form field reads

$$
\begin{aligned}
\kappa_{D}^{2} \rho(\varphi, \phi)= & \kappa_{D}^{2} f_{1}^{2} \exp \left(\frac{2 d_{1}-D}{\sqrt{(D-1)(D-2)}} \phi\right) \\
& \times \exp \left(2 \varphi \sqrt{\frac{d_{1}\left(D_{0}-2\right)}{D-2}}\right) .
\end{aligned}
$$

For brevity of notation, we introduce

$$
\begin{gathered}
a:=2 \sqrt{\frac{D_{0}-2}{d_{1}(D-2)}}, \quad b:=2 \sqrt{\frac{d_{1}}{(D-2)\left(D_{0}-2\right)}}, \\
c:=\frac{2 d_{1}-D}{\sqrt{(D-1)(D-2)}}, \quad h:=\kappa_{D}^{2} f_{1}^{2}
\end{gathered}
$$

so that the effective potential reads

$$
U_{e f f}=e^{b \varphi}\left[-\frac{1}{2} R_{1} e^{a \varphi}+U(\phi)+h e^{c \phi} e^{a d_{1} \varphi}\right] .
$$

From Eq. (51) we see that a real-valued form field $f_{1}$ implies a non-negative $h=\kappa_{D}^{2} f_{1}^{2} \geqslant 0$. For the rest of the paper, we continue to work with dimensionless scalar fields $\varphi, \phi$ instead of passing to canonical ones (modulo $8 \pi$ ): $\tilde{\varphi}$ $=\varphi M_{P l(4)}, \widetilde{\phi}=\phi M_{P l(4)}$, and $\widetilde{U}_{e f f}=M_{P l(4)}^{2} U_{e f f}$. The restoration of the correct dimensionality is obvious. 
In order to ensure a stabilization and asymptotical freezing of the internal space $M_{1}$, the effective potential should have a minimum with respect to the scalar field $\varphi$,

$$
\left.\partial_{\varphi} U_{\text {eff }}\right|_{\text {extr }}=0,
$$

so that for a minimum position at $\varphi_{0}=0$ (which corresponds to a compactification scale $\beta^{1}=0$ at the present time) it should hold

$$
\frac{a+b}{2} R_{1}=b U(\phi)+\left(a d_{1}+b\right) h e^{c \phi} .
$$

This formula shows that the potential $U_{e f f}(\varphi, \phi)$ must also have a minimum with respect to $\phi$, because without stabilization of $\phi$ the right hand side remains a dynamical function whereas the left hand side is a constant. This second extremum condition

$$
\left.\partial_{\phi} U_{e f f}\right|_{\text {extr }}=0
$$

yields

$$
\left.\left[\partial_{\phi} U+h c e^{c \phi}\right]\right|_{e x t r}=0 .
$$

Additionally, the eigenvalues of the mass matrix of the coupled $(\varphi, \phi)$-field system, i.e., the Hessian of the effective potential at the minimum position,

$$
J:=\left.\left(\begin{array}{ll}
\partial_{\varphi \varphi}^{2} U_{e f f} & \partial_{\varphi \phi}^{2} U_{e f f} \\
\partial_{\phi \varphi}^{2} U_{e f f} & \partial_{\phi \phi}^{2} U_{e f f}
\end{array}\right)\right|_{\text {extr }},
$$

should be positive definite,

$$
m_{1,2}^{2}=\frac{1}{2}\left[\operatorname{Tr}(J) \pm \sqrt{\operatorname{Tr}^{2}(J)-4 \operatorname{det}(J)}\right]>0 .
$$

According to the Sylvester criterion, this is equivalent to the conditions

$$
J_{11}>0, \quad J_{22}>0, \quad \operatorname{det}(J)>0 .
$$

From Eq. (57) we see that in the special case of $\left.\partial_{\varphi \phi}^{2} U_{\text {eff }}\right|_{\text {extr }}=0$ the Hessian is diagonal and the excitation modes of the fields decouple. The eigenvalues of $J$ coincide in this case with the masses squared of the scale factor excitations (gravitational excitons [22]) $m_{1}^{2}=m_{\varphi}^{2}$ and the excitations of the nonlinearity field $m_{2}^{2}=m_{\phi}^{2}$.

Let us now analyze the stability conditions (54), (56), and (59) explicitly. For this purpose we introduce the auxiliary notations

$$
\phi_{0}:=\left.\phi\right|_{e x t r}, \quad X:=e^{A \phi_{0}} \geqslant 0, \quad q:=8 \alpha \Lambda_{D}
$$

and rewrite the potentials $U, U_{\text {eff }}$, and the derivatives of $U_{e f f}$ at a possible minimum position $\left(\varphi_{0}=0, \phi_{0}\right)$ as

$$
\left.U_{0} \equiv U\right|_{e x t r}=\frac{1}{8 \alpha} X^{-D /(D-2)}\left[(X-1)^{2}+q\right],
$$

$$
\begin{aligned}
\left.U_{\text {eff }}\right|_{\text {extr }}= & -\frac{1}{2} R_{1}+U_{0}(X)+h X^{\left(2 d_{1}-D\right) /(D-2)}, \\
\left.\partial_{\varphi} U_{\text {eff }}\right|_{\text {extr }}= & -\frac{a+b}{2} R_{1}+b U_{0}(X)+\left(d_{1} a+b\right) \\
& \times h X^{\left(2 d_{1}-D\right) /(D-2)}=0,
\end{aligned}
$$

$$
\begin{aligned}
\left.\partial_{\phi} U_{e f f}\right|_{\text {extr }}= & \frac{1}{8 \alpha} X^{-D /(D-2)}\left[(2 A-B) X^{2}-2(A-B) X\right. \\
& -(q+1) B]+h c X^{\left(2 d_{1}-D\right) /(D-2)}=0, \\
\left.\partial_{\varphi \varphi}^{2} U_{e f f}\right|_{\text {extr }}= & -\frac{a^{2}-b^{2}}{2} R_{1}-b^{2} U_{0}(X)+\left[\left(d_{1} a\right)^{2}-b^{2}\right] \\
& \times h X^{\left(2 d_{1}-D\right) /(D-2)}, \\
\left.\partial_{\varphi \phi}^{2} U_{e f f}\right|_{\text {extr }}= & c d_{1} a h X^{\left(2 d_{1}-D\right) /(D-2)},
\end{aligned}
$$

$$
\begin{aligned}
\left.\partial_{\phi \phi}^{2} U_{\text {eff }}\right|_{\text {extr }}= & \frac{1}{8 \alpha} X^{-D /(D-2)}\left[(2 A-B)^{2} X^{2}-2(A-B)^{2} X\right. \\
& \left.+(q+1) B^{2}\right]+c^{2} h X^{\left(2 d_{1}-D\right) /(D-2)}
\end{aligned}
$$

[The constants $A, B$ are defined in Eqs. (20) and (28), respectively.] We see that, for fixed dimensions $D_{0}$ and $d_{1}$, the two equations (63),(64) describe a three-dimensional algebraic variety $\mathcal{V} \subset \mathcal{M}$ in the five-dimensional parameter (moduli) space $^{9} \mathcal{M}=\mathrm{R}^{3} \times \mathbb{R}_{+}^{2} \ni\left(\alpha, \Lambda_{D}, R_{1}, h, X\right)$. On the variety, inequalities (59) of the Sylvester criterion define then the parameter region $\Upsilon \subset \mathcal{V}$ of stable compactifications. A natural strategy for extracting detailed information about the location of this stability region would consist in solving Eq. (64) for $X$ with subsequent back-substitution of the found roots into the inequalities (59) and Eq. (63). In the following consideration we restrict our attention to the three simplest nontrivial cases which are easy to handle analytically.

\section{A. Zero effective cosmological constant: $\Lambda_{e f f} \equiv 0$}

By definition, we have $\left.\Lambda_{\text {eff }} \equiv U_{\text {eff }}\right|_{\text {extr }}$ so that in the particular case $\Lambda_{e f f} \equiv 0$, Eq. (62) yields the additional constraint

$$
\left.U_{e f f}\right|_{e x t r}=-\frac{1}{2} R_{1}+U_{0}(X)+h X^{\left(2 d_{1}-D\right) /(D-2)}=0 .
$$

Combining this constraint with Eq. (63), we obtain the relation

$$
R_{1}=2 d_{1} h X^{\left(2 d_{1}-D\right) /(D-2)}=\frac{2 d_{1}}{d_{1}-1} U_{0}(X)
$$

\footnotetext{
${ }^{9}$ The compactification scale (modulus) $r_{1}$ of the internal space $M_{1}$ enters $\mathcal{V} \subset \mathcal{M}$ via curvature scalar $R_{1}$ [see Eq. (37)].
} 
which can be used to eliminate the $h X^{\left(2 d_{1}-D\right) /(D-2)}$ term from Eq. (64). As a result, we arrive at a simple quadratic equation in $X$ with physically sensible solutions,

$$
e^{A \phi_{0}} \equiv X=\left\{\begin{array}{l}
\frac{-1+\sqrt{1+\left(d_{1}-2\right) d_{1}(1+q)}}{d_{1}-2}, \quad d_{1}>2, \\
1+q, \quad d_{1}=2 .
\end{array}\right.
$$

With the help of Eqs. (69), (70) and repeated use of a substitution-elimination technique, the potential $U_{0}$ and the second derivatives (65)-(67) of the effective potential can be rewritten in the simpler form

$$
U_{0}(X)=\frac{d_{1}-1}{4 \alpha d_{1}} X^{-2 /(D-2)}(X-1)
$$

and

$$
\begin{aligned}
J_{11} & \left.\equiv \partial_{\varphi \varphi}^{2} U_{\text {eff }}\right|_{\text {extr }}=a^{2} d_{1} U_{0}(X), \\
J_{22} & \left.\equiv \partial_{\phi \phi}^{2} U_{\text {eff }}\right|_{\text {extr }} \\
& =\frac{B^{2}}{4 \alpha D^{2}} X^{-2 /(D-2)}\left[E X+4\left(D_{0}-1\right)\right], \\
J_{12} & \left.\equiv \partial_{\varphi \phi}^{2} U_{\text {eff }}\right|_{\text {extr }}=\frac{c d_{1} a}{d_{1}-1} U_{0}(X),
\end{aligned}
$$

where $E \equiv(D-4)^{2}+4\left(d_{1}-2\right)>0$ for $d_{1} \geqslant 2$. For the determinant of the Hessian (57) we get

$$
\begin{aligned}
\operatorname{det}(J)= & \frac{1}{\alpha\left(d_{1}-1\right)} \frac{D_{0}-2}{D-1} \\
& \times U_{0}(X) X^{-2 /(D-2)}\left[\left(d_{1}-2\right) X+1\right] .
\end{aligned}
$$

With Eqs. (69)-(75) at hand, we are well prepared to explicitly describe the location of the stability region $Y$ in the parameter space $\mathcal{M}$. Let us start with relation (69). From the non-negativity conditions $h \geqslant 0$ and $e^{A \phi_{0}} \equiv X \geqslant 0$ we immediately conclude that for stable spaces $M_{1}$ it should hold $R_{1} \geqslant 0$ and $U_{0}(X) \geqslant 0$. Furthermore, we see from the latter condition and the Sylvester criterion $J_{22}>0$, $\operatorname{det}(J)>0$ [applied to Eqs. (73) and (75)] that for internal spaces of dimension $d_{1} \geqslant 2$ the parameter $\alpha$ is restricted to positive values ${ }^{10}$ $\alpha>0$ (the limiting case $\alpha \rightarrow 0$ we discuss below). Finally, we note that Eq. (71) together with $U_{0}(X) \geqslant 0$ and $\alpha>0$ implies $X \geqslant 1$ and, hence, we find the following for $d_{1}>2$ and $d_{1}$ $=2$ from the roots (70): $q \equiv 8 \alpha \Lambda_{D} \geqslant 0$ and also $\Lambda_{D} \geqslant 0$.

\footnotetext{
${ }^{10}$ Obviously, a negative $\alpha$ would yield a maximum of the effective potential $U_{e f f}$ instead of a minimum and our model would become unstable with respect to the conformal excitations of the internal space. The condition $\alpha>0$ is also required in other $R^{2}$ models [48] to ensure tachyon-free configurations.
}

Summarizing the obtained restrictions, we can describe the part $\Theta$ of the parameter space $\mathcal{M}$ where the stability region $Y$ of the variety $\mathcal{V}$ is located:

$$
Y \subset \mathcal{V} \cap \Theta \subset \Theta=\left(\alpha \geqslant 0, \Lambda_{D} \geqslant 0, R_{1} \geqslant 0, h \geqslant 0, X \geqslant 1\right) \subset \mathcal{M} .
$$

It remains to clarify what happens in the various limiting cases when the parameters reach the boundary $\partial \Theta$.

(L.1.1) $q \rightarrow+0$. According to Eq. (70), this limit implies $X \rightarrow 1, \phi_{0} \rightarrow 0$. Because of $q=8 \alpha \Lambda_{D}$, we have to distinguish the two cases $\alpha \rightarrow 0$ and $\Lambda_{D} \rightarrow 0$. In these limits we obtain $U_{0}(X) \rightarrow \Lambda_{D}$ and $U_{0}(X) \rightarrow 0$, respectively.

(L.1.2) $\alpha \rightarrow+0, \Lambda_{D} \neq 0$. The case $\alpha \rightarrow 0$ describes the transition to a linear model. Here we have $U(\phi) \rightarrow \Lambda_{D}, R_{1}$ $\rightarrow\left[2 d_{1} /\left(d_{1}-1\right)\right] \Lambda_{D}$, and $\Lambda_{D} \rightarrow\left(d_{1}-1\right) h$. In this limit, the mass of the $\phi$-field excitations tends to infinity $m_{2}^{2} \rightarrow m_{\phi}^{2}$ $\rightarrow J_{22} \rightarrow \infty$ and the field itself becomes frozen at the position $\phi_{0} \rightarrow 0$. The stabilization of the internal space occurs for $R_{1}, h, \Lambda_{D}>0$ with the gravexciton masses $m_{1}^{2} \rightarrow m_{\varphi}^{2} \rightarrow J_{11}$ $=4\left[\left(D_{0}-2\right) /(D-2)\right] \Lambda_{D}$. This is in accordance with the results of Ref. [22], where a linear model with monopole terms was considered.

(L.1.3) $\Lambda_{D} \rightarrow 0, \alpha \neq 0$. Due to Eq. (70) and (L.1.1) this limit implies $X \rightarrow 1, \phi_{0} \rightarrow 0$, and $U_{0}(X) \rightarrow 0$ so that according to Eq. (74) the excitation masses $m_{1}, m_{2}$ decouple $\left(J_{12}\right.$ $\rightarrow 0)$ and the gravexciton mass vanishes $m_{1}^{2} \rightarrow m_{\varphi}^{2} \rightarrow 0$. Hence, the limit $\Lambda_{D} \rightarrow 0$ is connected with a destabilization of the internal space $M_{1}$. The mass of the nonlinearity field excitations $m_{\phi}$ remains finite, $m_{2}^{2} \rightarrow m_{\phi}^{2} \sim 1 / \alpha$ for $\alpha>0$.

(L.1.4) $h \rightarrow+0, \alpha \neq 0$. From Eqs. (69)-(71) we see that this limit of a vanishing form field is connected with $R_{1}$, $U_{0}(X), \Lambda_{D} \rightarrow+0$. Thus the excitations of the nonlinearity field $\phi$ decouple from gravexcitons $\left(J_{12} \rightarrow 0\right)$. Simultaneously, because of $R_{1} \rightarrow+0 \Rightarrow r_{1} \rightarrow \infty$, the internal space $M_{1}$ undergoes a decompactification and due to $U_{0}(X) \rightarrow 0$ the effective potential $U_{\text {eff }}$ becomes flat in the $\varphi$ direction $\left(J_{11} \rightarrow 0 \Rightarrow m_{\varphi} \rightarrow 0\right)$. This means that the internal space destabilizes, whereas $U_{\text {eff }}$ remains well behaved with respect to $\phi$. These results completely confirm the conclusions of paper [25] for a nonlinear gravitational model without form fields where a stabilization is only possible for $\Lambda_{\text {eff }}<0$.

Finally, we note that for a model with $d_{1}=D_{0}$ (and, hence, a vanishing trace of the form field EMT) the excitations of nonlinearity field $\phi$ decouple from the gravexcitons: $J_{12}=0$ because of $c=0$ in Eqs. (51), (74).

\section{B. Traceless EMT of the form field: $d_{1}=D_{0}$}

The easy handling of nonlinear models with a traceless form-field EMT as well as of models with a two-dimensional internal space $M_{1}$ is connected with the structure of Eq. (64). For $X>0, \alpha \neq 0$ we obtain from Eqs. (51),(64) and the definitions of $A$ and $B$

$$
\begin{array}{r}
\frac{1}{8 \alpha}\left[(D-4) X^{2}+4 X-(q+1) D\right] \\
+\left(2 d_{1}-D\right) h X^{2 d_{1} /(D-2)}=0 .
\end{array}
$$


This algebraic equation reduces to a simple quadratic equation in $X$ either when the last term vanishes due to $2 d_{1}-D$ $\equiv d_{1}-D_{0}=0$ (the case of a traceless form field EMT) or when its degree $l\left(d_{1}\right):=2 d_{1} /(D-2)$ equals 0,1 , or 2 . For $D_{0}=4$, we have $l\left(d_{1}=0\right)=0, l\left(d_{1}=2\right)=1$, and $l\left(d_{1} \rightarrow \infty\right)$ $\rightarrow 2$ so that only the model with $D_{0}=4, d_{1}=2$ is of physical interest. It will be the subject of Sec. III C.

For $d_{1}=D_{0}=4$, we find a physically sensible solution of Eq. (77),

$$
X=\frac{1}{2}(\sqrt{9+8 q}-1)
$$

We use this solution as well as the extremum condition (63) to rewrite Eqs. (61) $-(63)$ and (65) $-(67)$ in the simpler form

$$
\begin{aligned}
U_{0}(X) & =\frac{3}{16 \alpha} X^{-1 / 3}(X-1), \\
\Lambda_{\text {eff }}(X) & =\frac{1}{3} U_{0}(X)-h, \\
R_{1} & =4\left[\frac{1}{3} U_{0}(X)+h\right], \\
J_{11} & =\left.\partial_{\varphi \varphi}^{2} U_{\text {eff }}\right|_{\text {extr }}=\frac{2}{3}\left[9 h-U_{0}(X)\right], \\
J_{22} & =\left.\partial_{\phi \phi}^{2} U_{\text {eff }}\right|_{\text {extr }}=\frac{1}{14 \alpha} X^{-1 / 3}(2 X+1), \\
J_{12} & =\left.\partial_{\varphi \phi}^{2} U_{\text {eff }}\right|_{\text {extr }}=0 .
\end{aligned}
$$

Obviously, there is no mixing of the excitations of the nonlinearity field $\phi$ with gravexcitons $\left(J_{12}=0\right)$ in this case: $m_{\varphi}^{2}=J_{11}, m_{\phi}^{2}=J_{22}$. Further, we read off from $J_{22}>0, X$ $\geqslant 0$ that stable internal spaces are again only possible for $\alpha>0$ and from Eq. (78) and $X \geqslant 0$ that $q$ is restricted to the half-line $q \geqslant-1$. Additional information can be extracted by combining the condition $J_{11}>0$ with relations (80),(81), which gives

$$
16 h>R_{1}>16 U_{0}(X) / 9>8 \Lambda_{e f f} .
$$

For the realistic case of a positive effective cosmological constant we find according to Eqs. (80), (85) the conditions

$$
\Lambda_{e f f}>0: \quad h>R_{1} / 16>U_{0}(X) / 9>h / 3>0,
$$

and, hence, from Eqs. (78),(79) also the implication $U_{0}(X)$ $>0 \Rightarrow X>1 \Rightarrow q>0$. We therefore conclude that such configurations are only possible for internal spaces with positive scalar curvature $R_{1}>0$ and positive bulk cosmological constant $\Lambda_{D}>0$.

Let us briefly comment on some limiting cases.

(L.2.1) $h \rightarrow+0$. In this case we recover the result of [25] that stable configurations are only possible for $R_{1}, \Lambda_{D}, \Lambda_{\text {eff }}$ $<0$ [see the inequality chain (85)].
(L.2.2) $\alpha \rightarrow+0, \Lambda_{D} \neq 0$. For this transition to the linear model with freezing of the nonlinearity field at $\phi_{0} \rightarrow 0$ and diverging excitation mass $m_{\phi}^{2} \rightarrow \infty$, the stability sector $\Theta \subset \mathcal{M}$ can be read off from Eq. (85) via substitution $U_{0}(X) \rightarrow \Lambda_{D}$.

(L.2.3) $\Lambda_{D} \rightarrow 0, \alpha \neq 0$. The limit is connected with $q$ $\rightarrow 0, X \rightarrow 1, \phi_{0} \rightarrow 0, U_{0}(X) \rightarrow 0$ and we have to distinguish two special cases. For a nonvanishing form field strength $h$ $\neq 0$ according to Eqs. (82),(83) inequalities $J_{11}, J_{22}>0$ hold so that both excitation masses remain finite. For vanishing field strength $h \rightarrow+0$ we obtain $R_{1} \rightarrow 0, J_{11} \rightarrow 0, m_{1}^{2}=m_{\varphi}^{2}$ $\rightarrow 0$ and the internal space $M_{1}$ undergoes a destabilization/ decompactification with $r_{1} \rightarrow \infty$.

\section{Two-dimensional internal spaces: $d_{1}=2$}

According to Eqs. (64),(77), the extremum condition $\left.\partial_{\phi} U_{\text {eff }}\right|_{\text {extr }}=0$ for models with two-dimensional internal space $M_{1}$, and $D_{0}=4$ can be reduced to a quadratic equation and, hence, allows for an easy analytical handling of the models. Introducing the notation

$$
z:=4 \alpha h,
$$

Eq. (77) reads

$$
X^{2}-2(z-1) X-3(q+1)=0
$$

and has solutions

$$
X_{1,2}=z-1 \pm \sqrt{(z-1)^{2}+3(q+1)} .
$$

Furthermore, Eq. (77) can be used to simplify the elements of the Hessian $J$. Setting $D_{0}=4$ and $d_{1}=2$ everywhere in Eqs. (51),(65)-(67) and eliminating $q$ with the help of Eq. (88), we obtain ${ }^{11}$

$$
\begin{aligned}
& \left.J_{11} \equiv \partial_{\varphi \varphi}^{2} U_{\text {eff }}\right|_{\text {extr }}=\frac{1}{6 \alpha} X^{-1 / 2}(5 z+1-X), \\
& \left.J_{22} \equiv \partial_{\phi \phi}^{2} U_{e f f}\right|_{\text {extr }}=\frac{1}{10 \alpha} X^{-1 / 2}(X-z+1), \\
& \left.J_{12} \equiv \partial_{\varphi \phi}^{2} U_{\text {eff }}\right|_{\text {extr }}=-\frac{1}{2 \sqrt{5} \alpha} X^{-1 / 2} z,
\end{aligned}
$$

as well as

$$
\operatorname{det}(J)=-\frac{1}{60 \alpha^{2} X}\left(X^{2}-6 z X+8 z^{2}-4 z-1\right)
$$

It is now easy to describe the part $\Theta$ of the parameter space $\mathcal{M}$ where the stability region $Y \subset \mathcal{V}$ is located. We start by substituting the solutions (89) into $J_{22}$. Taking into account that $X \geqslant 0$, we get from the condition $J_{22}>0$ for $X_{1}, X_{2}$

\footnotetext{
${ }^{11}$ The curvature term in $J_{11}$ of Eq. (65) cancels because of $a=b$ $=1$ for $D_{0}=4, d_{1}=2$.
} 


$$
\pm \sqrt{(z-1)^{2}+3(q+1)} / \alpha>0 .
$$

Thus, the roots $X_{1}$ and $X_{2}$ correspond to $\alpha>0$ and $\alpha<0$, respectively. Because of $z=4 \alpha h, h \geqslant 0$ this leads to negative values for $X_{2}$ so that this root is unphysical and stable configurations are restricted to $X=X_{1}(z, q)$ and $\alpha>0$. The limiting case $\alpha \rightarrow+0$ will be considered separately below.

Furthermore, we see from the structure of Eqs. (90)-(93) that the Sylvester criterion selects a region $\Theta_{(z, X)}$ from the $(z, X)$ plane which can be interpreted as the projection of the stability region $Y$ on this plane. Explicitly we have

$$
\begin{aligned}
J_{11}>0 & \Rightarrow X<5 z+1, \\
J_{22}>0 & \Rightarrow X>z-1, \\
\operatorname{det}(J)>0 & \Rightarrow\left\{\begin{array}{l}
X<3 z+\sqrt{z^{2}+4 z+1} \\
X>3 z-\sqrt{z^{2}+4 z+1},
\end{array}\right.
\end{aligned}
$$

where the inequalities (97) are easily derived from Eq. (93) by calculating the critical values $X_{c}(z)$ for which $\operatorname{det}\left(J\left[z, X_{c}(z)\right]\right)=0$. The intersection $\Theta_{(z, X)}$ of the sectors defined by the conditions (95)-(97) and $X>0, z \geqslant 0$ is shown in Fig. 1.

In order to obtain information about the values of $q$ $=8 \alpha \Lambda_{D}$ (and $\Lambda_{D}$ ) which allow for a stable internal space $M_{1}$, it proves convenient to map the region $\Theta_{(z, X)}$ via the quadratic equation (88) or its solution $X_{1}(z, q)$ from the $(z, X)$ plane on an equivalent region $\Theta_{(z, q)}$ of the $(z, q)$ plane. For this purpose it is sufficient to transform the inequalities (95)-(97) and $X \geqslant 0, z \geqslant 0$ for $X$ and $z$ into an equivalent inequality set for $q$ and $z$. Let us demonstrate the mapping, e.g., for inequality (95). Substituting $X=X_{1}(z, q)$ $=z-1+\sqrt{(z-1)^{2}+3(q+1)}$ into the equation for the critical line $X=X_{c}(z)=5 z+1$ and solving for $q$, we obtain as an image of this line $X_{c}(z)$ a corresponding critical curve

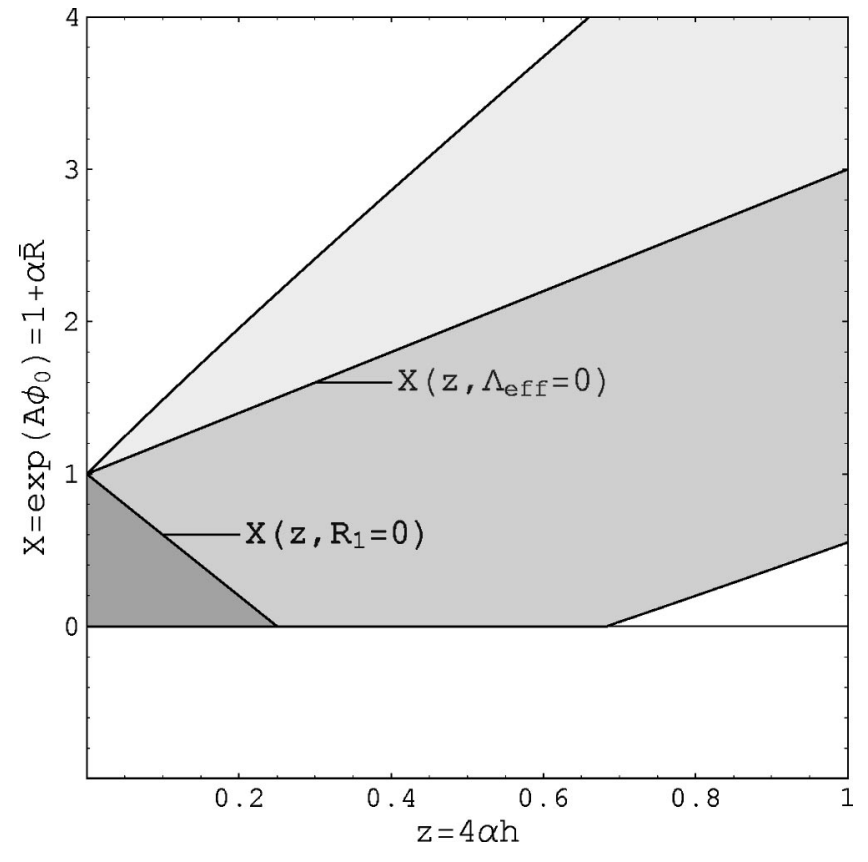

FIG. 1. Projection $\Theta_{(z, X)}$ of the stability region $Y \subset \mathcal{V} \subset \mathcal{M}$ on the $(z, X)$ plane (shaded areas). The two lines $\Lambda_{\text {eff }}=0$ and $R_{1}=0$ [given in Eqs. (104)] separate the stable regions with $[X$ $\left.>X\left(z, \Lambda_{e f f}=0\right):\left(\Lambda_{e f f}>0, R_{1}>0\right)\right],\left[X\left(z, \Lambda_{e f f}=0\right)>X>X\left(z, R_{1}\right.\right.$ $\left.=0):\left(\Lambda_{e f f}<0, R_{1}>0\right)\right]$ and $\left[X<X\left(z, R_{1}=0\right):\left(\Lambda_{e f f}<0, R_{1}\right.\right.$ $<0)]$.

$q_{c}(z)=z(5 z+6)$ on the $(z, q)$ plane. [The same curve can be obtained by substitution of $X_{c}(z)$ into the quadratic equation (88).] With the help of two test points $P_{1}=\left[z_{1}, q_{1}\right.$ $\left.>q_{c}\left(z_{1}\right)\right], P_{2}=\left[z_{2}, q_{2}<q_{c}\left(z_{2}\right)\right]$ above and below the critical curve $q_{c}(z)$, e.g., $P_{1}=(1,26), P_{2}=(2,0)$, it is then easily seen that $X_{1}(z, q)<5 z+1$ maps into $q<z(5 z+6)$. Applying the same technique to Eqs. (96),(97) we obtain

$$
\begin{aligned}
J_{11}>0 & \Rightarrow q<z(5 z+6), \\
J_{22}>0 & \Rightarrow q>-1-\frac{1}{3}(z-1)^{2}, \\
\operatorname{det}(J)>0 & \Rightarrow\left\{\begin{array}{l}
q<-1+\left[4 z^{2}+10 z+1+2(2 z+1) \sqrt{z^{2}+4 z+1}\right] / 3, \\
q>-1+\left[4 z^{2}+10 z+1-2(2 z+1) \sqrt{z^{2}+4 z+1}\right] / 3 .
\end{array}\right.
\end{aligned}
$$

Additionally we find from $X \geqslant 0$

$$
\begin{array}{ll}
q \geqslant-1-\frac{1}{3}(z-1)^{2} & \text { for } \quad z \geqslant 1, \\
q \geqslant-1 & \text { for } \quad 0 \leqslant z \leqslant 1 .
\end{array}
$$

The resulting intersection region $\Theta_{(z, q)}$ of Eqs. (98)-(101) is depicted in Fig. 2.

Let us now turn to the scalar curvature $R_{1}$ and the fourdimensional effective cosmological constant $\Lambda_{\text {eff }}$ $=\left.U_{\text {eff }}\right|_{\text {extr }}$. The structure of Eqs. (61),(62), and (63) suggests to consider $R_{1}$ and $\Lambda_{e f f}$ as functions of $(z, X, q, \alpha)$. Eliminating $q$ from Eqs. (62), (63) [with the help of (88)], we obtain 


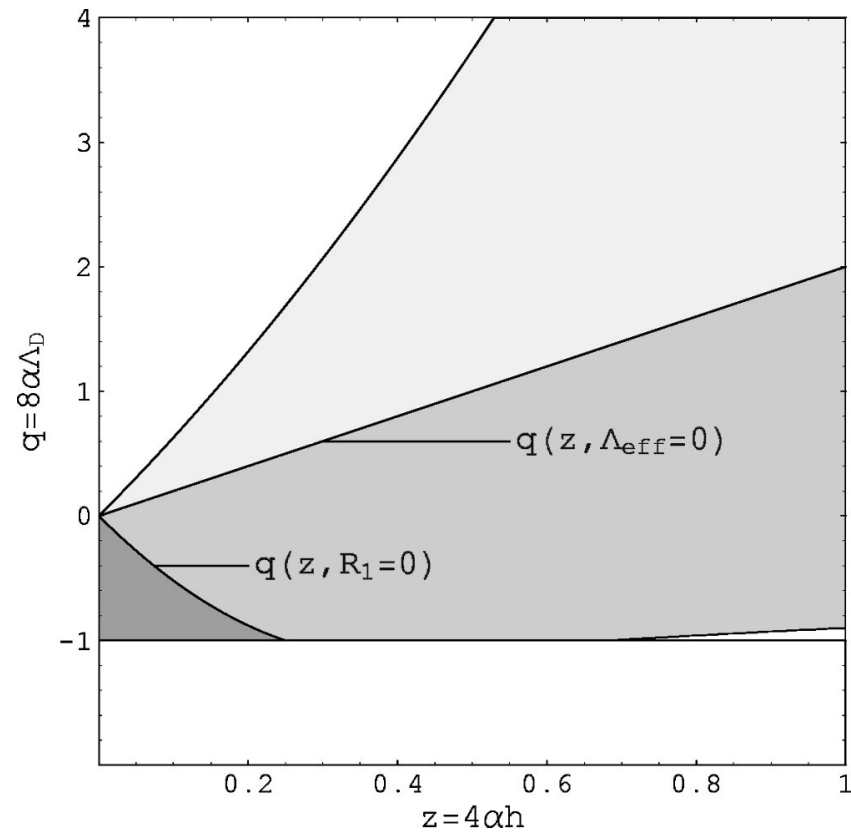

FIG. 2. Projection $\Theta_{(z, q)}$ of the stability region $Y \subset \mathcal{V} \subset \mathcal{M}$ on the $(z, q)$ plane (shaded areas). The two lines $\Lambda_{\text {eff }}=0$ and $R_{1}=0$ [given in Eqs. (105)] separate the stable regions with $[q$ $\left.>q\left(z, \Lambda_{e f f}=0\right):\left(\Lambda_{e f f}>0, R_{1}>0\right)\right],\left[q\left(z, \Lambda_{e f f}=0\right)>q>q\left(z, R_{1}\right.\right.$ $\left.=0):\left(\Lambda_{e f f}<0, R_{1}>0\right)\right]$ and $\left[q<q\left(z, R_{1}=0\right):\left(\Lambda_{e f f}<0, R_{1}\right.\right.$ $<0)]$.

$$
\begin{gathered}
R_{1}=\frac{1}{6 \alpha} X^{-1 / 2}(X+4 z-1), \\
\Lambda_{e f f}=\frac{1}{12 \alpha} X^{-1 / 2}(X-2 z-1) .
\end{gathered}
$$

The graphics of the functions

$$
\begin{gathered}
R_{1}(z, X)=\left.0 \Rightarrow X\right|_{\left(R_{1}=0\right)}=1-4 z, \\
\Lambda_{e f f}(z, X)=\left.0 \Rightarrow X\right|_{\left(\Lambda_{e f f}=0\right)}=1+2 z
\end{gathered}
$$

are included in Fig. 1. For completeness, we map them also on the $(z, q)$ plane. Following the same scheme as above, we obtain

$$
\begin{gathered}
R_{1}(z, q)=\left.0 \Rightarrow q\right|_{\left(R_{1}=0\right)}=2 z(4 z-3), \quad 0 \leqslant z<1 / 4 \\
\Lambda_{e f f}=\left.0 \Rightarrow q\right|_{\left(\Lambda_{e f f}=0\right)}=2 z
\end{gathered}
$$

and the correspondences

$$
\begin{aligned}
& R_{1}>0 \text { for } X>1-4 z, \quad q>2 z(4 z-3) \text {, } \\
& \Lambda_{e f f}>0 \text { for } X>1+2 z, \quad q>2 z .
\end{aligned}
$$

From Fig. 1 and Fig. 2 we see that the nonlinear model with two-dimensional internal space $M_{1}$ allows for stable configurations only in the cases

$$
\begin{aligned}
& \Lambda_{e f f} \geqslant 0 \text { for } R_{1}>0 \\
& \Lambda_{e f f}<0 \text { for } \operatorname{sgn}\left(R_{1}\right)= \pm 1,0
\end{aligned}
$$

It contains no stable configurations with an accelerated expansion of the Universe $\left(\Lambda_{e f f}>0\right)$ for internal spaces of negative or vanishing scalar curvature $R_{1}$.

Finally, we comment on some limiting cases.

(L.3.1) $h \rightarrow+0, q \neq 0$. According to Figs. 1 and 2 this limit corresponds to a vanishing form field $z \rightarrow+0$, and a stabilization is possible in the case of $R_{1}<0$. Furthermore, for $z \rightarrow 0$ we can approximate $X=X_{1}(z, q) \approx(v-1)(1$ $+z / v)$ with $v:=\sqrt{4+3 q}>1$ and the masses of the normal excitation modes of the coupled $(\varphi-\phi)$ field system follow from Eqs. (58),(90)-(92) as

$$
\begin{aligned}
& m_{1}^{2}=\frac{1}{6 \alpha}(v-1)^{-1 / 2}\left[2-v+\frac{9}{2} z+O\left(z^{2}\right)\right], \\
& m_{2}^{2}=\frac{1}{10 \alpha}(v-1)^{-1 / 2}\left[v-\frac{2+v}{2 v} z+O\left(z^{2}\right)\right] .
\end{aligned}
$$

In the special case $z=0$ we completely reproduce our earlier results [25] on nonlinear stabilized models without form fields $\left(d_{1}=2\right): \quad m_{\varphi}^{2}=m_{1}^{2}=-U_{0}(X), \quad m_{\phi}^{2}=m_{2}^{2}=X^{-1 / 2}(X$ $+1) /(10 \alpha)$.

(L.3.2) $\alpha \rightarrow+0 ; \Lambda_{D}, h \neq 0$. For this transition to a linear model we have as in (L.1.1) $U(X) \rightarrow \Lambda_{D}$ as well as a freezing of the nonlinearity field at $\phi_{0} \rightarrow 0, X \rightarrow 1$. Using the approximation

$$
\begin{aligned}
X & =X_{1}(z, q) \\
& =1+z+(3 q-2 z) / 4-(3 q-2 z)^{2} / 64+z^{2} / 4+O\left(\alpha^{3}\right)
\end{aligned}
$$

we obtain the excitation masses as

$$
\begin{aligned}
& m_{\phi}^{2} \rightarrow m_{1}^{2}=\frac{\alpha^{-1}-2 h}{5}+O(\alpha) \rightarrow \infty, \\
& m_{\varphi}^{2} \rightarrow m_{2}^{2}=3 h-\Lambda_{D}+O(\alpha)>0
\end{aligned}
$$

so that the freezing is clearly seen from the diverging mass of the nonlinearity field. Additionally, we find from Eqs. (102),(103)

$$
\begin{array}{r}
R_{1}=\Lambda_{D}+3 h-\frac{\alpha}{6}\left[27\left(h+\Lambda_{D}\right)^{2}-8 h^{2}\right]+O\left(\alpha^{2}\right) \\
\Lambda_{e f f}=\frac{\Lambda_{D}-h}{2}+\frac{3}{4} \alpha\left[\left(h+\Lambda_{D}\right)^{2}-4 \Lambda_{D}^{2}\right]+O\left(\alpha^{2}\right),
\end{array}
$$

which in the special case of a vanishing effective cosmological constant $\Lambda_{e f f}=0$ reproduces the results of Ref. [22] for a linear model with a Freund-Rubin form field: $h=\Lambda_{D}$ $=R_{1} / 4, m_{\varphi}^{2}=2 h$.

(L.3.3) $\Lambda_{D} \rightarrow 0 ; \alpha, h \neq 0$. In this case we have $q \rightarrow 0$. A substitution of the approximation $X=X_{1}(z, q)=z-1+[(z$ $\left.-1)^{2}+3\right]^{1 / 2}+3\left[(z-1)^{2}+3\right]^{-1 / 2} q / 2-O\left(q^{2}\right)$ into the Hes- 
sian shows that there is no special behavior of the excitation masses connected with this limit.

(L.3.4) $\Lambda_{D}, h \rightarrow 0, \alpha \neq 0$. From Eq. (90) it follows in this limit $J_{11} \rightarrow 0$, so that beside a decoupling of the excitations the gravexciton mass vanishes $m_{1}^{2} \rightarrow m_{\varphi}^{2} \rightarrow 0$ and the internal space $M_{1}$ destabilizes. This is in full agreement with [25] where a stabilization for $h=0$ requires $\Lambda_{D}<0$.

(L.3.5) $R_{1} \rightarrow 0$. In the limit $R_{1} \rightarrow 0$ one observes a regular behavior similar to (L.3.3). For parameter points near the line $X_{0}(z):=X_{\left(R_{1}=0\right)}(z)=1-4 z, 0 \leqslant z<1 / 4$ we find from Eq. (102) $X=X_{0}(z)+6 \alpha R_{1} X_{0}^{1 / 2}+O\left(\alpha^{2} R_{1}^{2}\right)$ so that the Hessian yields excitation masses of the form $m_{1,2}^{2}\left(R_{1} \approx 0\right)$ $=m_{1,2}^{2}\left(R_{1}=0, z\right)+\sigma_{1,2}(z) \alpha R_{1}+O\left(\alpha^{2} R_{1}^{2}\right)$ with some regular coefficients $\sigma_{1,2}(z)$ and

$$
\begin{aligned}
m_{1,2}^{2}\left(R_{1}=\right. & 0, z)=\frac{1}{40 \alpha} X_{0}^{-1 / 2}\left[9-5 X_{0}\right. \\
& \left.\mp \sqrt{4\left(3-5 X_{0}\right)^{2}+5\left(X_{0}-1\right)^{2}}\right] .
\end{aligned}
$$

The masses $m_{1,2}^{2}\left(R_{1}=0, z\right)$ have finite values except at the limiting points $X_{0}(z \rightarrow 1 / 4) \rightarrow 0$ (or $\left.\phi_{0} \rightarrow-\infty\right)$ and $X_{0}(\alpha$ $\rightarrow 0) \rightarrow 1$ (or $\phi_{0} \rightarrow 0$ ), where both or one of the masses diverge. We see that, with the exception of the limiting point ${ }^{12}$ $X_{0}(\alpha \neq 0) \rightarrow 1$, there occurs no destabilization of the internal space $M_{1}$ for vanishing scalar curvatures $R_{1}$. Due to the smooth behavior of the excitation masses under the transition $R_{1} \rightarrow 0$ we can identify this limit with a stable decompactification $r_{1} \rightarrow \infty$ of an internal space $M_{1}$ with fixed topology. Clearly, in our local approach a stable decompactified space with $r_{1} \rightarrow \infty$ is indistinguishable from a stabilized internal space which is Ricci-flat from the very beginning.

\section{CONCLUSIONS AND DISCUSSION}

In the present paper we investigated multidimensional gravitational models with a non-Einsteinian form of the action. In particular, we assumed that the action is an arbitrary smooth function of the scalar curvature $f(R)$. Additionally, the $D$-dimensional spacetime was endowed with solitonic form fields of generalized block-orthogonal Freund-Rubin type. This bulk matter ansatz leads to a naturally factorized geometry and a spontaneous compactification can be associated with it. For the considered models, we concentrated on the stabilization problem for the extra dimensions. As a technique we used a reduction of the nonlinear gravitational model to a linear one with an additional self-interacting scalar field (nonlinearity scalar field). The factorized geometry as well as the generalized Freund-Rubin ansatz for the solitonic form field allowed for a dimensional reduction of the considered models and a transition to the Einstein frame. As a result, we obtained an effective four-dimensional model

\footnotetext{
${ }^{12}$ According to Eq. (113), the limit $X_{0}(\alpha \neq 0) \rightarrow 1$ gives $m_{1}^{2}$ $\rightarrow m_{\varphi}^{2} \rightarrow 0, m_{2}^{2} \rightarrow m_{\phi}^{2} \rightarrow 1 /(5 \alpha)$. On the other hand, $X_{0}(\alpha \neq 0) \rightarrow 1$ implies $z=4 \alpha h \rightarrow 0$ and according to Eq. (89) also $\Lambda_{D} \rightarrow 0$. Thus, the results of (L.3.1) and (L.3.4) can be used to reproduce the same behavior of the excitation masses via Eq. (108).
}

with nonlinearity scalar field and additional minimally coupled scalar fields which describe conformal excitations of the scale factors of the internal space.

A detailed stability analysis was carried out for the three simplest configurations of a model with one internal factor space $M_{1}$ and a quadratic curvature term: $f(R)=R+\alpha R^{2}$ $-2 \Lambda_{D}$, where $\Lambda_{D}$ plays the role of a $D$-dimensional bare (bulk) cosmological constant. These three configurations are characterized respectively by (1) a vanishing fourdimensional effective cosmological constant $\Lambda_{\text {eff }}$, (2) a traceless form-field EMT, or (3) a $\left(d_{1}=2\right)$-dimensional internal factor space $M_{1}$. For all three configurations, a stabilization of the internal space is only possible in the case of a non-negative nonlinearity parameter $\alpha \geqslant 0$ and a bulk cosmological constant $\Lambda_{D}$ restricted by the condition $q \equiv 8 \alpha \Lambda_{D}>$ -1 . The transition $\left(\Lambda_{D} \rightarrow 0, h \rightarrow 0\right)$ is connected with a decompactification $\left(R_{1} \rightarrow 0, r_{1} \rightarrow \infty\right)$ of the internal space $M_{1}$. At the same time, it leads to a flattening of the effective potential in the direction of the scale factor excitations and, hence, to a destabilization of $M_{1}$ (for a similar limiting behavior, see also Ref. [25]).

From the three configurations, the model with the twodimensional internal space shows the richest features. It allows for stable configurations in the cases $\left(\Lambda_{\text {eff }} \geqslant 0, R_{1}>0\right)$ and $\left(\Lambda_{\text {eff }}<0\right.$, any sign of $\left.R_{1}\right)$ as well as for Ricci-flat internal spaces $\left(R_{1}=0\right)$. Interestingly, the various stable configurations belong to a connected region in the parameter space $\mathcal{M}$ and one can smoothly pass from one type of configuration to another one, including a transition to stable Ricci-flat internal spaces which can be described as "stable decompactifications:" $R_{1} \rightarrow 0, r_{1} \rightarrow \infty$. As pointed out in the Introduction, such a rich picture became possible due to the presence of the real-valued form fields which satisfy the NEC and the WEC and which compete with the nonlinearity scalar field. The latter satisfies the NEC only marginally and can violate the WEC.

Interestingly, for $\left(d_{1}=2\right)$-dimensional internal spaces there exist parameter configurations with $\alpha, \Lambda_{D}, h, R_{1}>0$ that can provide positive values of the effective fourdimensional cosmological constant $\Lambda_{e f f}>0$ [see, e.g., Eqs. (106),(107)]. Thus, an accelerated expansion of the Universe seems possible in accordance with observational data. Let us assume that the values of the bulk cosmological constant $\Lambda_{D}$ and the form field strength $h$ are set at some characteristic scale $\Lambda_{D} \sim h \sim \bar{M}^{2}$. Then we find for the parameters $q$ $\sim 8 \alpha \bar{M}^{2}, \quad z \sim 4 \alpha \bar{M}^{2}$, and, hence, $q \sim 2 z$. The latter corresponds to $X \sim 1+2 z \sim 1+8 \alpha \bar{M}^{2}$ [see Eq. (89)] and comparison with (105) shows that such configurations should yield an almost vanishing effective cosmological constant $\Lambda_{\text {eff }}$ $\sim 0$. With the help of Eq. (102) we can estimate the scalar curvature $R_{1}$ of the internal space as

$$
R_{1} \sim \frac{z}{\alpha} X^{-1 / 2} \sim \frac{1}{\alpha} \frac{z}{\sqrt{1+2 z}} \sim \frac{4 \bar{M}^{2}}{\sqrt{1+8 \alpha \bar{M}^{2}}} .
$$


On the other hand, its value is connected with the fundamental scales $M_{*\left(4+d_{1}\right)}, M_{P l(4)}$ by the relations (1), (2), and (37):

$$
R_{1} \sim r_{1}^{-2} \sim\left(\frac{M_{*\left(4+d_{1}\right)}}{M_{P l(4)}}\right)^{4 / d_{1}} M_{*\left(4+d_{1}\right)}^{2} .
$$

As mentioned in the discussion after Eq. (35), the value of $X$ can be used as a measure of the nonlinearity of the original model: $\alpha \bar{R}=e^{A \phi_{0}}-1 \equiv X-1$. We see that weakly nonlinear configurations correspond to $X \approx 1$, whereas $X \gg 1$ leads to a strongly nonlinear regime. With the help of Eqs. (114) and (115) we express this dimensionless nonlinearity parameter $X$ in terms of the different scales contained in our model:

$$
X \sim 1+8 \alpha \bar{M}^{2} \sim 16\left(\frac{\bar{M}}{M_{*\left(4+d_{1}\right)}}\right)^{4}\left(\frac{M_{P l(4)}}{M_{*\left(4+d_{1}\right)}}\right)^{8 / d_{1}} .
$$

From Eq. (116) we see that setting $\bar{M} \sim M_{*\left(4+d_{1}\right)}$ we obtain $X \gg 1$ for ADD-type $\mathrm{TeV}$-scale models whereas $X \sim 1$ can only be achieved for standard KK models with $M_{P l(4)}$ $\sim M_{*\left(4+d_{1}\right)}$. Stably compactified internal spaces in ADDtype models can be obtained within a weakly nonlinear regime $X \sim 1$ if the bulk cosmological constant $\Lambda_{D}$ and the form field strength $h$ are related with the fundamental scales as

$$
\begin{aligned}
& \Lambda_{D} \sim h \sim \bar{M}^{2}, \\
& \bar{M} \sim \frac{1}{2} M_{*\left(4+d_{1}\right)}\left(\frac{M_{*\left(4+d_{1}\right)}}{M_{P l(4)}}\right)^{2 / d_{1}} .
\end{aligned}
$$

For $M_{*\left(4+d_{1}\right)} \sim 1-30 \mathrm{TeV}$ and $d_{1}=2$ this implies $\bar{M}$ $\sim 10^{-4}-10^{-1} \mathrm{eV}$. It is interesting to note that this mass scale is of the same order as the lowest possible supersymmetry breaking scale $m \sim M_{S U S Y}^{2} / M_{P l(4)}$ in the minimal supersymmetric extension of the standard model (MSSM) [49] with $M_{S U S Y} \sim 1 \mathrm{TeV}$.

Above, we demonstrated that the assumption $\Lambda_{D} \sim h$ can result in a small effective cosmological constant $\Lambda_{\text {eff }}$. Let us now estimate the relation between $\Lambda_{e f f}$ and $\Lambda_{D}, h$ in more detail and compare it with the observable value ${ }^{13}$ of $\Lambda_{\text {eff }}$ $\sim 10^{-123} \Lambda_{P l(4)}$. For simplicity, we will restrict our consideration to a weak nonlinearity regime with $X \approx 1, \alpha \gtrsim 0$, where the approximations (111) and (112) of (L.3.2) are valid. From Eq. (112), we see that to ensure a sufficiently small $\Lambda_{\text {eff }}$ the bulk cosmological constant $\Lambda_{D}$ and the field strength $h$ of the solitonic form field should be connected by

$$
h=(1+\epsilon) \Lambda_{D} .
$$

\footnotetext{
${ }^{13}$ In our normalization conventions holds $c=\hbar=1$ and $\Lambda_{P l(4)}$ $\sim M_{P l(4)}^{2} \sim L_{P l(4)}^{-2}$.
}

The value of $\epsilon$ can be estimated as follows. With the help of relations (111) and (117), we find

$$
\begin{gathered}
10^{-123} \Lambda_{P l(4)} \sim \Lambda_{e f f} \approx \epsilon \Lambda_{D}\left(1-6 \alpha \Lambda_{D}\right) / 2, \\
\Lambda_{D} \sim \bar{M}^{2} \sim R_{1}
\end{gathered}
$$

and, hence,

$$
10^{-123} \sim \epsilon\left(\frac{M_{*\left(4+d_{1}\right)}}{M_{P l(4)}}\right)^{4 / d_{1}+2}
$$

so that

$$
\epsilon \sim 10^{-65}
$$

for $d_{1}=2, M_{*\left(4+d_{1}\right)} \sim 30 \mathrm{TeV}$. According to Eq. (119), this value of $\epsilon$ is not sensitive to changes of the nonlinearity parameter $\alpha$ in a weakly nonlinear curvature regime. Thus, we arrive at the conclusion that the ADD scenario in its simplest extended version can provide a simultaneous stabilization of the extra dimensions together with an adjustment of the effective cosmological constant to its observed value only in the case of a strong fine tuning. Although the solitonic form fields of our model are located in the compactified extra dimensions, the tuning of their effective energy density $h$ to the bulk cosmological constant $\Lambda_{D}, h=(1+\epsilon) \Lambda_{D}$, is of a similar type as the four-form-tuning discussed in Weinberg's no-go theorem [40] for a resolution of the cosmological constant problem (CCP). A shifting of the CCP to a parameter fine tuning is a rather general feature of models with compactified additional dimensions and form fields. ${ }^{14}$ In a slightly reshaped form it also appears in the recently proposed brane-world model with two-dimensional "football"shaped large extra dimensions [51] (see also [52]) where the adjustment of the on-brane cosmological constant is shifted to an adjustment of the parameters of the off-brane "football." A possible resolution of the CCP for similar higherdimensional models with form fields following from an M-theory setup was presented in Ref. [53]. Proposals for a resolution of the CCP within other scenarios comprise various anthropic approaches [50,54], shifting of the CCP to a singularity problem [55], possible graviton compositeness [56], a holographic approach [57] as well as nonlocal modifications of gravity [58]. However, there is still no satisfactory and comprehensive solution of the CCP. The problem will probably continue to challenge the scientific community until a final understanding of quantum gravity is achieved.

\section{ACKNOWLEDGMENTS}

U.G. and A.Z. thank H. Nicolai and the Albert Einstein Institute, as well as the Department of Physics of the University of Beira Interior for their kind hospitality during the preparation of this paper. The work of A.Z. was supported by

\footnotetext{
${ }^{14}$ For a similar mechanism in RSII-type models with form fields, see Ref. [50].
} 
a BCC grant from CENTRA-IST and partly supported by the program SCOPES (Scientific co-operation between Eastern Europe and Switzerland) of the Swiss National Science Foundation, project No. 7SUPJ062239. U.G. acknowledges support from DFG grant KON/1344/2001/GU/522. Additionally, this research work was partially supported by the grants POCTI/32327/P/FIS/2000, CERN/P/FIS/43717/2001, and CERN/P/FIS/43737/2001.
[1] M.B. Green, J.H. Schwarz, and E. Witten, Superstring Theory (Cambridge University Press, Cambridge, England, 1987); J. Polchinski, String Theory (Cambridge University Press, Cambridge, England, 1998).

[2] J. Polchinski, Phys. Rev. Lett. 75, 4724 (1995).

[3] N. Arkani-Hamed, S. Dimopoulos, and G. Dvali, Phys. Lett. B 429, 263 (1998).

[4] I. Antoniadis, N. Arkani-Hamed, S. Dimopoulos, and G. Dvali, Phys. Lett. B 436, 257 (1998).

[5] N. Arkani-Hamed, S. Dimopoulos, and G.J. March-Russell, Phys. Rev. D 63, 064020 (2001).

[6] C.D. Hoyle et al., Phys. Rev. Lett. 86, 1418 (2001); G. Dvali, G. Gabadadze, X. Hou, and E. Sefusatti, Phys. Rev. D 67, 044019 (2003).

[7] L. Randall and R. Sundrum, Phys. Rev. Lett. 83, 4690 (1999).

[8] G. Dvali, G. Gabadadze, and M. Porrati, Phys. Lett. B 485, 208 (2000).

[9] N. Arkani-Hamed, S. Dimopoulos, and G. Dvali, Phys. Rev. D 59, 086004 (1999).

[10] V.A. Rubakov, Usp. Fiz. Nauk 171, 913 (2001) [Phys. Usp. 44, 871 (2001)].

[11] L. Randall and R. Sundrum, Phys. Rev. Lett. 83, 3370 (1999).

[12] U. Günther and A. Zhuk, Class. Quantum Grav. 18, 1441 (2001)

[13] J.M. Cline and J. Vinet, Phys. Rev. D 68, 025015 (2003).

[14] L. Wang, R.R. Caldwell, J.P. Ostriker, and P.J. Steinhardt, Astrophys. J. 530, 17 (2000).

[15] T. Banks, M. Dine, and A.E. Nelson, J. High Energy Phys. 06, 014 (1999).

[16] N. Arkani-Hamed, S. Dimopoulos, N. Kaloper, and J. MarchRussell, Nucl. Phys. B567, 189 (2000).

[17] S.M. Carroll, J. Geddes, M.B. Hoffman, and R.M. Wald, Phys. Rev. D 66, 024036 (2002).

[18] J. Geddes, Phys. Rev. D 65, 104015 (2002).

[19] D.A. Demir and M. Shifman, Phys. Rev. D 65, 104002 (2002).

[20] S. Nasri, P.J. Silva, G.D. Starkman, and M. Trodden, Phys. Rev. D 66, 045029 (2002).

[21] L. Perivolaropoulos and C. Sourdis, Phys. Rev. D 66, 084018 (2002); L. Perivolaropoulos, ibid. 67, 123516 (2003).

[22] U. Günther and A. Zhuk, Phys. Rev. D 56, 6391 (1997).

[23] U. Günther and A. Zhuk, Stable Compactification and Gravitational Excitons from Extra Dimensions, Proceedings Workshop Modern Modified Theories of Gravitation and Cosmology, Beer Sheva, Israel, 1997 [Hadronic J. 21, 279 (1998)]; U. Günther, S. Kriskiv, and A. Zhuk, Gravitation Cosmol. 4, 1 (1998); U. Günther and A. Zhuk, Class. Quantum Grav. 15, 2025 (1998).

[24] U. Günther and A. Zhuk, Phys. Rev. D 61, 124001 (2000).

[25] U. Günther, P. Moniz, and A. Zhuk, Phys. Rev. D 66, 044014 (2002).

[26] R. Kerner, Gen. Relativ. Gravit. 14, 453 (1982); J.D. Barrow and A.C. Ottewill, J. Phys. A 10, 2757 (1983); J.P. Duruisseu and R. Kerner, Gen. Relativ. Gravit. 15, 797 (1983); B. Whitt, Phys. Lett. 145B, 176 (1984); J.D. Barrow and S. Cotsakis, Phys. Lett. B 214, 515 (1988); K. Maeda, J.A. Stein-Schabes, and T. Futamase, Phys. Rev. D 39, 2848 (1989); G. Magnano and L.M. Sokolowski, ibid. 50, 5039 (1994); D. Wands, Class. Quantum Grav. 11, 269 (1994).

[27] K. Maeda, Phys. Rev. D 39, 3159 (1989).

[28] J. Ellis, N. Kaloper, K.A. Olive, and J. Yokoyama, Phys. Rev. D 59, 103503 (1999).

[29] P.J.E. Peebles and B. Ratra, Rev. Mod. Phys. 75, 559 (2003).

[30] R. Bean, S.H. Hansen, and A. Melchiorri, Phys. Rev. D 64, 103508 (2001); R. Bean and A. Melchiorri, ibid. 65, 041302(R) (2002); R. Bean, S.H. Hansen, and A. Melchiorri, Nucl. Phys. B (Proc. Suppl.) 110, 167 (2002).

[31] T.D. Saini, S. Raychaudhury, V. Sahni, and A. Starobinsky, Phys. Rev. Lett. 85, 1162 (2000).

[32] K.S. Stelle, in High Energy Physics and Cosmology, 1996 Summer School, ICTP, Trieste, Italy, 1996, edited by E. Gava et al. (World Scientific, Singapore, 1997), pp. 287-339.

[33] P.G.O. Freund and M.A. Rubin, Phys. Lett. 97B, 233 (1980).

[34] D.L. Wiltshire, Phys. Rev. D 36, 1634 (1987).

[35] A. Lukas, B.A. Ovrut, and D. Waldram, Nucl. Phys. B495, 365 (1997); B509, 169 (1998).

[36] V.D. Ivashchuk and V.N. Melnikov, Class. Quantum Grav. 18, R1 (2001).

[37] J. Gray and E.J. Copeland, J. High Energy Phys. 06, 046 (2001).

[38] C. Barcelo and M. Visser, Int. J. Mod. Phys. D 11, 1553 (2002).

[39] A. Dolgov, in The Very Early Universe, edited by G. Gibbons, S. Hawking, and S. Siklos (Cambridge University Press, Cambridge, England, 1983), pp. 449-458.

[40] S. Weinberg, Rev. Mod. Phys. 61, 1 (1989).

[41] V. Sahni and A. Starobinsky, Int. J. Mod. Phys. D 9, 373 (2000).

[42] N. Straumann, "On the cosmological constant problems and the astronomical evidence for a homogeneous energy density with negative pressure," astro-ph/0203330.

[43] A. Hindawi, B.A. Ovrut, and D. Waldram, Prog. Theor. Phys. Suppl. 123, 397 (1996); E. Kiritsis and B. Pioline, Nucl. Phys. B508, 509 (1997); M.B. Green, M. Gutperle, and P. Vanhove, Phys. Lett. B 409, 177 (1997); C.P. Bachas, P. Bain, and M.B. Green, J. High Energy Phys. 05, 011 (1999); S. Nojiri and S. Odintsov, Phys. Rev. D 62, 064018 (2000).

[44] R.R. Caldwell, Phys. Lett. B 545, 23 (2002).

[45] V.D. Ivashchuk, V.N. Melnikov, and A.I. Zhuk, Nuovo Cimento Soc. Ital. Fis., B 104, 575 (1989).

[46] M. Rainer and A. Zhuk, Phys. Rev. D 54, 6186 (1996).

[47] D.D. Sokolov and V.F. Shvartsman, Sov. Phys. JETP 39, 196 
(1974); M. Lachieze-Rey and J.-P. Luminet, Phys. Rep. 254, 135 (1995); J.-P. Luminet and B.F. Roukema, "Topology of the universe: theory and observation," astro-ph/9901364.

[48] J. Audretsch, A. Economou, and C.O. Lousto, Phys. Rev. D 47, 3303 (1993).

[49] J. Schwarz and N. Seiberg, Rev. Mod. Phys. 71, S112 (1999); G. Dvali, G. Gabadadze, and M. Shifman, Mod. Phys. Lett. A 16, 513 (2001).

[50] B. Bajc and G. Gabadadze, Phys. Lett. B 474, 282 (2000).

[51] S. Carroll and M. Guica, "Sidestepping the cosmological constant with football-shaped extra dimensions," hep-th/0302067.

[52] I. Navarro, "Codimension two compactifications and the cosmological constant problem," hep-th/0302129.

[53] R. Bousso and J. Polchinski, J. High Energy Phys. 06, 006
(2000).

[54] J. Garriga and A. Vilenkin, Phys. Rev. D 64, 023517 (2001); R. Kallosh and A. Linde, ibid. 67, 023510 (2003).

[55] N. Arkani-Hamed, S. Dimopoulos, N. Kaloper, and R. Sundrum, Phys. Lett. B 480, 193 (2000); S. Kachru, M. Schulz, and E. Silverstein, Phys. Rev. D 62, 045021 (2000); J. Cline and H. Firouzjahi, ibid. 65, 043501 (2002).

[56] R. Sundrum, J. High Energy Phys. 07, 001 (1999).

[57] T. Banks, "Cosmological breaking of supersymmetry or little lambda goes back to the future II," hep-th/0007146.

[58] N. Arkani-Hamed, S. Dimopoulos, G. Dvali, and G. Gabadadze, "Non-local modification of gravity and the cosmological constant problem," hep-th/0209227. 